\title{
Posture compensation of a walking assistive device using zero-moment point to stabilize motions on stairs
}

\author{
Bo-Rong YANG*, Hee-Hyol LEE* and Eiichiro TANAKA* \\ *Graduate School of Information, Production and Systems, Waseda University \\ 2-7 Hibikino, Wakamatsu-ku, Kita-Kyushu, Fukuoka 808-0135, Japan \\ E-mail: tanakae@waseda.jp
}

Received: 5 June 2019; Revised: 2 September 2019; Accepted: 11 October 2019

\begin{abstract}
The main objective of this research was to stabilize the motions of a walking assistive device on the stairs. We proposed a posture-compensated method suitable for the stairs and proved the proposed theory in simulations and experiments. To adapt elderly people to daily life, an assistive device that could support the walking motion and protect the users from falls was utilized. Because the target users needed to walk on the stairs where they could easily fall, the device adjusted posture to stabilize users. Zero-moment point (ZMP) was used to quantify the stability of the device. To prevent collisions during the compensation process, the assistive device maintained the stride length because the width of the stairs is invariable. The target positions of the device feet were moved the same distance. The projection of gravity and the inertia force was kept in support polygons that varied for different walking phases to remain stable. Particularly, in simulation, the virtual slope method was quoted to calculate the location of ZMP. In experiments, pressure sensors were employed to measure ZMP. The results of the experiments showed that the assistive device, employing the proposed method, guided the user to successfully maintain the ZMP on the footplate. In conclusion, the proposed method is available for stabilizing various assistive devices, especially for slow-walking motions on stairs.
\end{abstract}

Keywords: Walking assistive device, Walking on stairs, Posture compensation, Zero-moment point, Supporting polygon

\section{Introduction}

Proper exercise is considered to prevent muscle loss in the elderly people and weakened patients. Therefore, various assistive devices are being developed to help target users perform long-duration repeated exercise that prevents their loss of muscle strength.

Elderly people and weakened patients who suffer from movement disorders chose to depend on wheelchairs for their mobility. Wheelchairs are convenient; however, they cannot provide weakened patients and elderly people with an opportunity to exercise (Tanaka et al., 2012). In the meantime, if they hardly ever walk or do some light exercise for a period of time, their muscle strength will decline, and their physical condition will become worse. Therefore, it is necessary to develop a solution that is helpful in enhancing their walking ability and preventing muscle atrophy. Some research showed that gait training is useful for enhancing physical ability (Reinkensmeyer et al., 2006). Hence, a walking assistive device that provides a recovery opportunity for elderly people and weakened patients is a better solution compared with a wheelchair.

Walking assistive apparatuses are widely utilized on some types of patients or elderly people to assist them in daily life activity. ReWalk ${ }^{\mathrm{TM}}$ assists the hip and knee joints of spinal cord injury patients (Talaty et al., 2013). This device can start from a sitting posture. After the user wears the device, he or she can begin to walk. A wearable power-assist locomotor (WPAL) uses a worm gear mechanism, the device has a compact spatial distribution and provides the user 
with a powerful assistive force (Hirano et al., 2015). The Hybrid Assistive Limb (HAL ${ }^{\circledR}$ ) was designed to help elderly people or patients who suffer from gait disorder to enhance their power (Suzuki et al., 2007).

However, most of the previous research is only applicable to flat ground, not stairs. For some elderly people and weakened patients, if they want to go sightseeing or visit certain places, they can rely on an electric bicycle or motorbike. As for places located on mountains, such as a temple or shrine, there is no tool that allows them to walk on the stairs. To solve this kind of issue, a device that can assist the user to walk on stairs is necessary.

Various walking conditions such as flat ground, stairs, and slopes have distinct acquirement for muscles (Kerrigan et al., 1998). Hence, exercise and walking assistive for these conditions are also important. While walking on stairs, safety is the most critical issue. Therefore, we utilized the zero-moment-point (ZMP) to judge whether the device and user are stable (Vukobratovic and Borovac, 2004). As a standard of stability, the ZMP needs to be controlled and maintained in the support polygon to prevent the user from stumbling. Generally, there are very many ZMP control methods. For example, there is preview control, which defines trajectories in advance and lets the real ZMP trace a predefined ZMP (Shimmyo et al., 2013). Although this method is applied widely on humanoid robots, it cannot ensure a constant stride length or humanoid walking trajectories. Because a robot traces target ZMP trajectories and not the foot trajectories of a human, this method is not suitable for walking assistive devices. Several walking assistive devices thus adopted other ZMP control methods. The stride-compensated ZMP control method is an example (Sakurai, 2012), which maintained the position of the ZMP in the support polygon by adjusting the stride length and walking cycle of the walking assistive apparatus. Compared with the preview control method, the predefined foot trajectories make the human walking motion more suitable than using predefined ZMP trajectories.

However, because the distance between every two stairs is constant, stride length cannot be adjusted on the stairs. Besides, because the walking motion on stairs is very slow, the walking speed and cycle are also difficult to change. Hence, some walking assistive devices utilize a posture-compensated method that mainly adjusts the position of the center of gravity (COG) to shift ZMP to maintain balance.

Low et al. (2006) proposed a method for controlling the ZMP by compensating the motion of the trunk. From the experiment results, the position of ZMP was kept in a stable area by controlling the angle between the upper body and lower limbs. However, for those walking assistive apparatuses for lower limb without a rotatable upper limb, this method is unsuitable. Therefore, Aphiratsakun and Parnichkun (2009) proposed a new ZMP control method that maintains balance by adjusting the angle of the ankle joint. In this research, a fuzzy logic control was used, but it had some problems. As for complex human performance, an assistive device with a fuzzy logic control method always lacks flexibility and users feel uncomfortable. Therefore, we proposed a new posture-compensated method that has better prospects. It simultaneously changes the target position of both legs to adjust the position of ZMP. It can be used not only on stairs but also on flat ground. The proposed method was achieved on a walking assistive device that can assist the whole lower legs. The appearance of the walking assistive device is shown in Fig. 1.

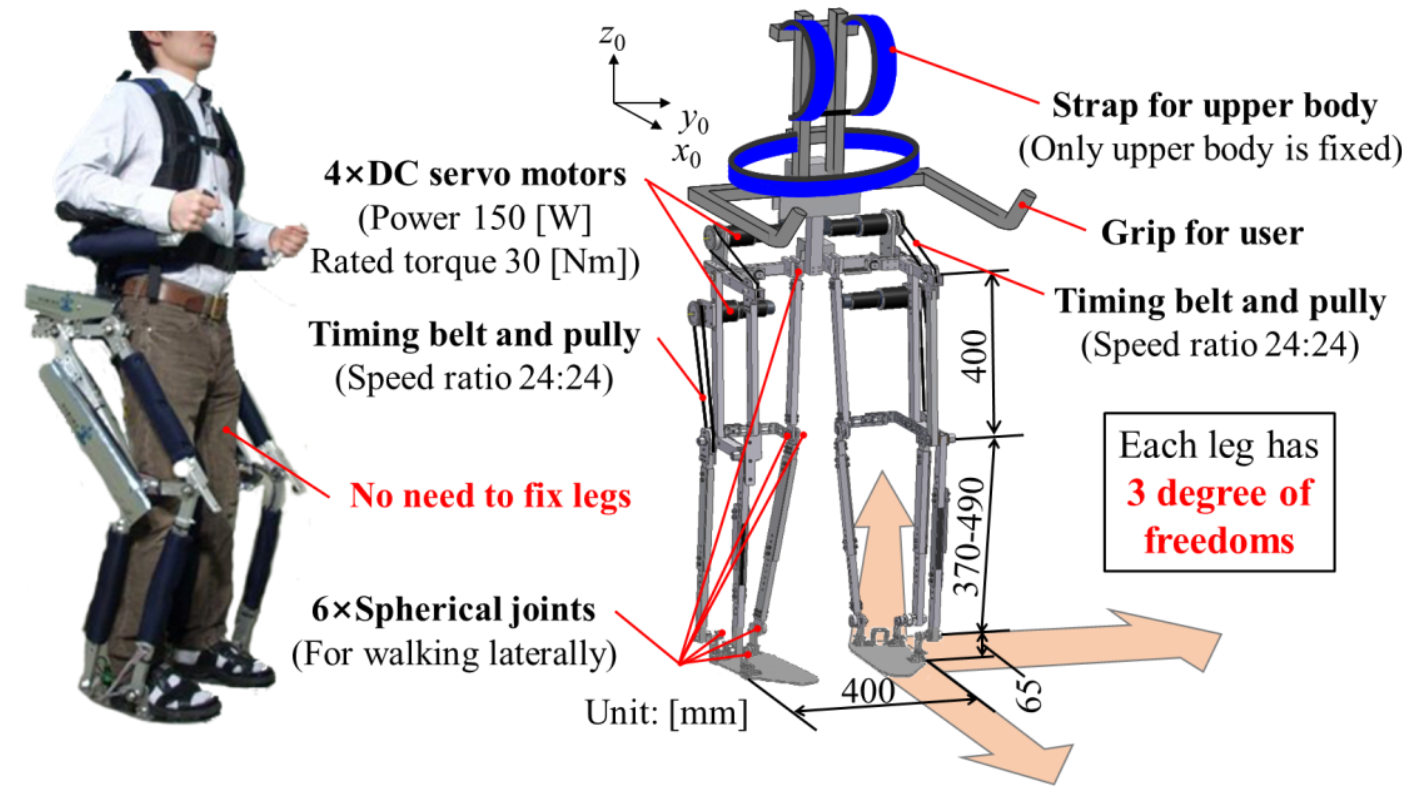

Fig. 1 Appearance of the walking assistive device. 
This paper is organized as follows: Section 2 describes the mechanical structure and dynamic model of the proposed assistive device. The principle of the posture-compensated method is described in Section 3. The study of the simulation is introduced in Section 4, including the calculation of ZMP. Finally, the proposed theory is verified through the experimental results in Section 5.

\section{The walking assistive device}

\subsection{Mechanical and controller system}

We used a very special mechanism called a spatial parallel link mechanism (Tanaka et al., 2011), as shown in Fig. 1. Using this mechanism, we could ensure that the sole of the foot structurally followed the flat steps. Compared with traditional apparatuses, this apparatus can assist the rotation of hip, knee, and ankle joints using only two motors (Maxon Re-40) for each leg. The total weight of the device is $23 \mathrm{~kg}$. With the gravity compensation function, each motor of the device can deliver enough power $(150 \mathrm{~W})$ to stand by itself. Thus, the user has enough assistance to walk smoothly. The gear head reduction ratio of the motors is $126: 1$. The rated torque of the motors is $30 \mathrm{Nm}$. Two timing belts whose velocity ratio is $24: 24$ were utilized on the device to drive the hip and knee joints. Additionally, the device used spherical joints to walk laterally.

The control system of the apparatus is illustrated in Fig. 2. The device utilizes the SH4 as a central processing unit and various kinds of sensors to detect the situations of the surroundings and the device itself. In detail, we selectively use pressure sensors for various purposes. As a trigger for attacking the next step, two of the pressure sensors are utilized to judge the departure of the foot. To judge whether the device is stable, four of the pressure sensors are utilized to measure the position of the ZMP, which acts as a stability index. Angle sensors are utilized to attain the posture of the device. The whole controller is supported by a lithium ion battery.

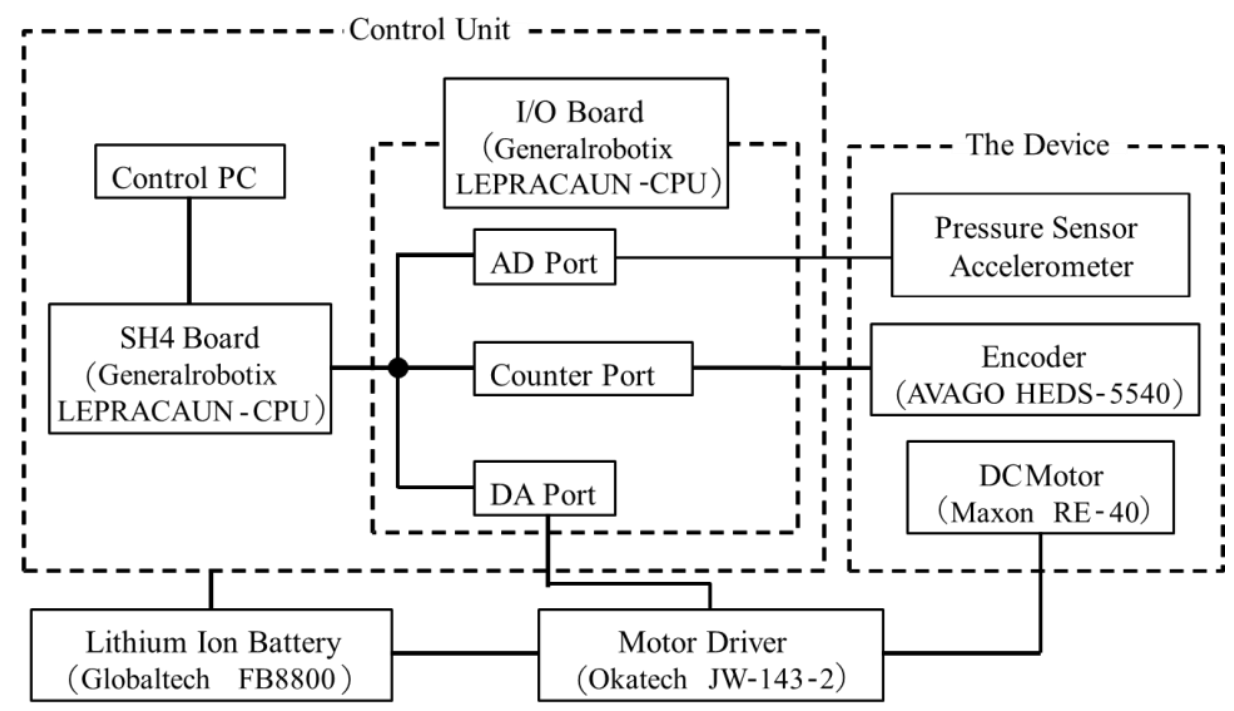

Fig. 2 General view of a control system.

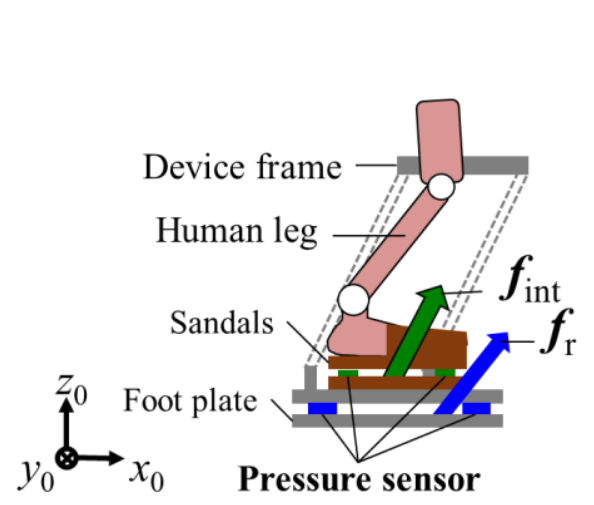

Location of sensors

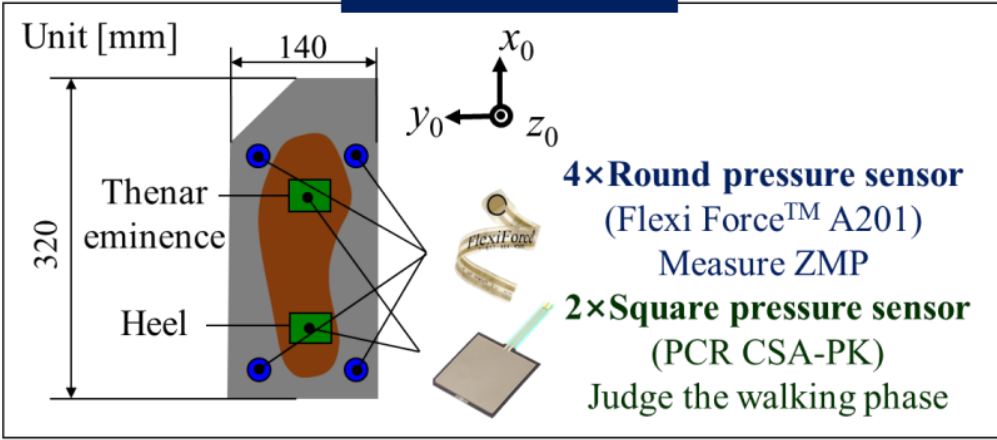

Fig. 3 Pressure sensor layout. 
The layout of the pressure sensors is shown in Fig. 3. Four piezoresistive force sensors, Flexi Force ${ }^{\mathrm{TM}}$ A201, which can measure up to $445 \mathrm{~N}$ are distributed at the four corners of the underside of each footplate to measure the location of the ZMP. These four sensors are clamped between two metal plates to be fixed tightly. Because the sensors were mounted under the footplate of the device, the sensors can measure the vertical component of the floor reaction force $f_{\mathrm{r}}$ of the user-exoskeleton system. From the value of the four round sensors, the location of the pressure center was gotten to indicate the stability of the entire system. The thickness of this type of sensor is $0.203 \mathrm{~mm}$. Additionally, the sensing area of this sensor is small ( $9.53 \mathrm{~mm}$ diameter). During walking, the pressure can be concentrated on four small areas. Therefore, the pressure center can be measured precisely. In addition, to estimate the walking phase of the user, two sensitive pressure sensors, PCR technical CSA-PK, are connected under the thenar eminence and heel of each user's foot. These sensors are also the trigger for judging whether the user wants to go ahead or not. The pressure sensitive sensors are distributed in the undersides of the soles of a user's sandals but above the footboard of the assistive device, as shown as the green squares on the left side of Fig. 3. Since the sensors were mounted above the footplate of the device, the force measured by square pressure sensors is only caused by the weight of the user. This force is also the vertical component of the interaction force $f_{\text {int }}$ between the user and shoes. With the two types of pressure sensors above, the controller can know the stability and walking phase of the assistive device. After that, the torque output from each actuator will also be adjusted accordingly.

\subsection{Dynamic mode}

Our proposed mechanism assists the user means of a parallel link mechanism. Using this mechanism, the sole of the device moves horizontally relative to the ground. The mechanism has two degrees of freedoms in the front and rear directions. The foot of the user is supported by the flat sole of the device.

To get the power required to allow the assistive device to assist the user, we calculated the output torque. The equations of dynamic torque for the human and the assistive device are determined by various physical parameters. The structure of the device is shown in Fig. 4(a). It can be modeled as a two-dimensional model, as shown in Fig. 4(b).

In the figure, $\tau_{1}$ and $\tau_{2}$ are the torques applied at the hip and knee joints of the assistive device, respectively. Meanwhile, $\theta_{1}$ and $\theta_{2}$ are the angles of the hip and knee joints. $\alpha_{w b}$ is the assistive ratio of the user that is smaller than 1. $l_{b}, l_{c}, l_{d}, l_{e}, l_{f}$, and $l_{h}$ are the respective lengths of each link. In addition, $m_{b}, m_{c}, m_{d}, m_{e}, m_{f}$, and $m_{h}$ denote the mass of each link, whereas $m_{\text {leg }}$ is the mass of the user's leg that is estimated to be $12.075 \mathrm{~kg}$. $m_{\text {leg }}$ was calculated according to the average ratio of one leg's mass to the whole human body's mass (Nakamura et al., 2003). We assumed that the mass of the whole user's body $m_{\text {human }}$ is $70 \mathrm{~kg}$. Hence, $m_{l e g}=0.1725 \times m_{\text {human }}=12.075 \mathrm{~kg} . l_{g b}, l_{g c}, l_{g d}, l_{g e}, l_{g f}$, and $l_{g h}$ represent the distance to the COG of each link. Finally, $\tilde{I}_{b}, \tilde{I}_{c}, \tilde{I}_{d}, \tilde{I}_{e}, \tilde{I}_{f}$, and $\tilde{I}_{h}$ are the inertia moments of each link. Some details of each parameter are in Table 1 (Tanaka et al., 2012). The length, distance of COG, mass and inertia moment are gotten from the 3D model of the device in Autodesk ${ }^{\circledR}$ Inventor $^{\circledR}$.

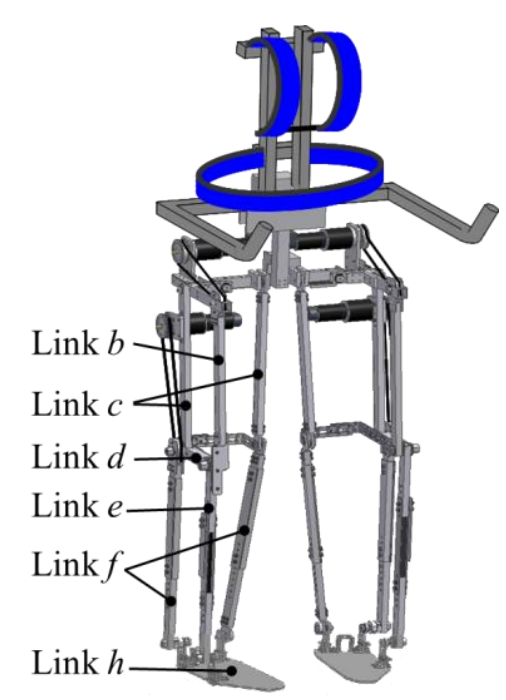

(a) Names of links for modeling

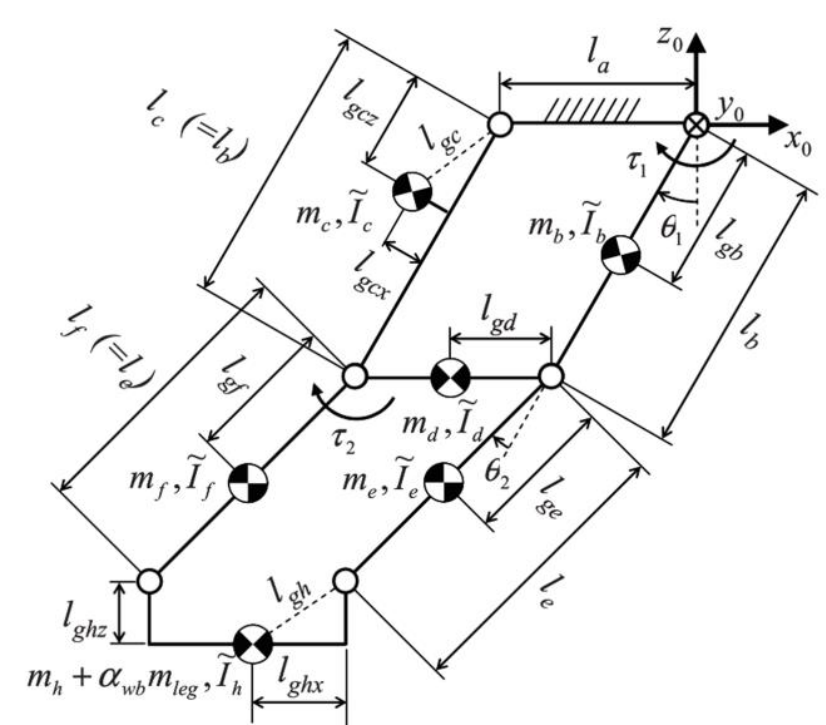

(b) Assistive device model to for equation (side view)

Fig. 4 Dynamic model of the assistive device. 
Table 1 Data of variables for each part of the assistive device.

\begin{tabular}{c|c}
\hline \hline$l_{b}[\mathrm{~m}]$ & 0.40 \\
\hline$l_{e}[\mathrm{~m}]$ & 0.49 \\
\hline$l_{g b}[\mathrm{~m}]$ & 0.20 \\
\hline$l_{g c x}[\mathrm{~m}]$ & 0.03 \\
\hline$l_{g c z}[\mathrm{~m}]$ & 0.12 \\
\hline$l_{g e}[\mathrm{~m}]$ & 0.24 \\
\hline$l_{g f}[\mathrm{~m}]$ & 0.19 \\
\hline$l_{g h x}[\mathrm{~m}]$ & 0.06 \\
\hline$l_{g h z}[\mathrm{~m}]$ & 0.04 \\
\hline$l_{g h}[\mathrm{~m}]$ & 0.07 \\
\hline
\end{tabular}

\begin{tabular}{c|c}
\hline \hline$m_{b}[\mathrm{~kg}]$ & 0.37 \\
\hline$m_{c}[\mathrm{~kg}]$ & 1.88 \\
\hline$m_{d}[\mathrm{~kg}]$ & 0.55 \\
\hline$m_{e}[\mathrm{~kg}]$ & 0.45 \\
\hline$m_{f}[\mathrm{~kg}]$ & 0.79 \\
\hline$m_{h}[\mathrm{~kg}]$ & 1.15 \\
\hline$\tilde{I}_{b}\left[\mathrm{kgm}^{2}\right]$ & 0.11 \\
\hline$\tilde{I}_{c}\left[\mathrm{kgm}^{2}\right]$ & 0.14 \\
\hline$\tilde{I}_{e}\left[\mathrm{kgm}^{2}\right]$ & 0.07 \\
\hline$\tilde{I}_{f}\left[\mathrm{kgm}^{2}\right]$ & 0.21 \\
\hline
\end{tabular}

We can derive the torque of each joint to drive one leg of the assistive device as shown in Eq. (1).

$$
\boldsymbol{\tau}=M(\boldsymbol{\theta}) \ddot{\boldsymbol{\theta}}+\boldsymbol{h}(\dot{\boldsymbol{\theta}}, \boldsymbol{\theta})+\boldsymbol{g}_{\text {ap }}(\boldsymbol{\theta})+\alpha_{\mathrm{gh}} \boldsymbol{g}_{\mathrm{h}}(\boldsymbol{\theta})-J^{\mathrm{T}}\left(\boldsymbol{f}_{\mathrm{r}}+\boldsymbol{f}_{\mathrm{p}}\right) \in \mathrm{R}^{2}
$$

Here, $\boldsymbol{\tau}$ and $\boldsymbol{\theta}$ are the torque and the angle of hip and knee joints as shown in Fig. 4(b).

$\boldsymbol{\tau}=\left\{\begin{array}{ll}\tau_{1} & \tau_{2}\end{array}\right\}^{\mathrm{T}} \in \mathrm{R}^{2}, \quad \boldsymbol{\theta}=\left\{\begin{array}{ll}\theta_{1} & \theta_{2}\end{array}\right\}^{\mathrm{T}} \in \mathrm{R}^{2}$.

$M$ is a $2 \times 2$ symmetric positive inertia matrix, representing the inertia moment of the human-exoskeleton system that the device needs to compensate. $\boldsymbol{h}$ describes the $2 \times 1$ Coriolis vector.

$M(\boldsymbol{\theta})=\left[\begin{array}{cc}I_{1}+I_{2}+2 R C_{2} & I_{2}+2 R C_{2} \\ I_{2}+2 R C_{2} & I_{2}\end{array}\right] \in \mathrm{R}^{2 \times 2}, \quad \boldsymbol{h}(\dot{\boldsymbol{\theta}}, \boldsymbol{\theta})=\left\{\begin{array}{c}-2 R S_{2} \dot{\theta}_{1} \dot{\theta}_{2}-R S_{2} \dot{\theta}_{2}^{2} \\ R S_{2} \dot{\theta}_{1}^{2}\end{array}\right\} \in \mathrm{R}^{2}$.

$I_{1}=m_{b} l_{g b}^{2}+m_{c} l_{g c}^{2}+\left(m_{d}+m_{e}+m_{f}+m_{h}+\alpha_{w b} m_{l e g}\right) l_{b}^{2}+\tilde{I}_{b}+\tilde{I}_{c}$,

$I_{2}=m_{e} l_{g e}^{2}+m_{f} l_{g f}^{2}+m_{h} l_{e}^{2}+\alpha_{w b} m_{l e g} l_{e}^{2}+\tilde{I}_{e}+\tilde{I}_{f}$,

$R=\left(m_{e} l_{g e}+m_{f} l_{g f}+m_{h} l_{e}+\alpha_{w b} m_{l e g} l_{e}\right) l_{b}$.

$\boldsymbol{g}_{\text {ap }}$ and $\boldsymbol{g}_{\mathrm{h}}$ denote $2 \times 1$ gravitational vector. $\boldsymbol{g}_{\text {ap }}$ describes the gravity compensation for one of the assistive device's leg. $\boldsymbol{g}_{\mathrm{h}}$ represents the gravity compensation for one of the user's leg.

$\boldsymbol{g}_{\text {ap }}(\boldsymbol{\theta})=\left\{\begin{array}{c}{\left[\left\{m_{b} l_{g b}+m_{c} l_{g c z}+\left(m_{d}+m_{e}+m_{f}+m_{h}\right) l_{b}\right\} S_{1}+\left(m_{e} l_{g e}+m_{f} l_{g f}+m_{h} l_{e}\right) S_{12}\right] g} \\ \left(m_{e} l_{g e}+m_{f} l_{g f}+m_{h} l_{e}\right) S_{12} g\end{array}\right\} \in \mathrm{R}^{2}$,

$\boldsymbol{g}_{\mathrm{h}}(\boldsymbol{\theta})=\left\{\begin{array}{c}\alpha_{\mathrm{gh}} \alpha_{w b}\left(m_{\text {leg }} l_{b} S_{1}+m_{\text {leg }} l_{e} S_{12}\right) g \\ \alpha_{\mathrm{gh}} \alpha_{w b} m_{l e g} l_{e} S_{12} g\end{array}\right\} \in \mathrm{R}^{2}$.

$J$ is the Jacobian matrix that converts the force or moment on the footplate to the hip and knee joints, $\quad J=\left[\begin{array}{lll}J_{1} & J_{2}\end{array}\right] \in \mathrm{R}^{6 \times 2}$. $J_{1} \in \mathrm{R}^{6}$ is the Jacobian matrix from the hip joint to the sole of the assistive device. $\boldsymbol{J}_{2} \in \mathrm{R}^{6}$ is the Jacobian matrix from the knee joint of the apparatus to the flat step.

$f_{\mathrm{r}}$ is the floor reaction force in the stance phase. In section 2.2, around sensor that can measure floor reaction force was introduced. However, the sensor can only be utilized as the judgment of stability. Because this kind of sensors can only measure the floor reaction force in the vertical direction, the horizontal forces are ignored. To calculate the torque that each motor needs to output precisely, the floor reaction forces in three directions $\left(f_{\mathrm{rx}}, f_{\mathrm{ry}}\right.$, and $\left.f_{\mathrm{rz}}\right)$ were calculated according to the three graphs in (Nakamura et al., 2003). The forces in three directions are expressed by ratio of human weight that is changed by walking phase. Therefore, according to the walking phase, the reaction forces in directions can be calculated.

$f_{\mathrm{p}}$ is the force generated by the assistive device that makes the foot of the assistive move along the predefined trajectory, as shown in Fig. 5. The trajectory was from a healthy person.

$\boldsymbol{f}_{\mathrm{r}}=\left\{\begin{array}{llllll}f_{\mathrm{rx}} & f_{\mathrm{ry}} & f_{\mathrm{rz}} & 0 & 0 & 0\end{array}\right\}^{\mathrm{T}} \in \mathrm{R}^{6}, \quad \boldsymbol{f}_{\mathrm{p}}=\left\{\begin{array}{llllll}f_{\mathrm{px}} & f_{\mathrm{py}} & f_{\mathrm{pz}} & 0 & 0 & 0\end{array}\right\}^{\mathrm{T}} \in \mathrm{R}^{6}$. 


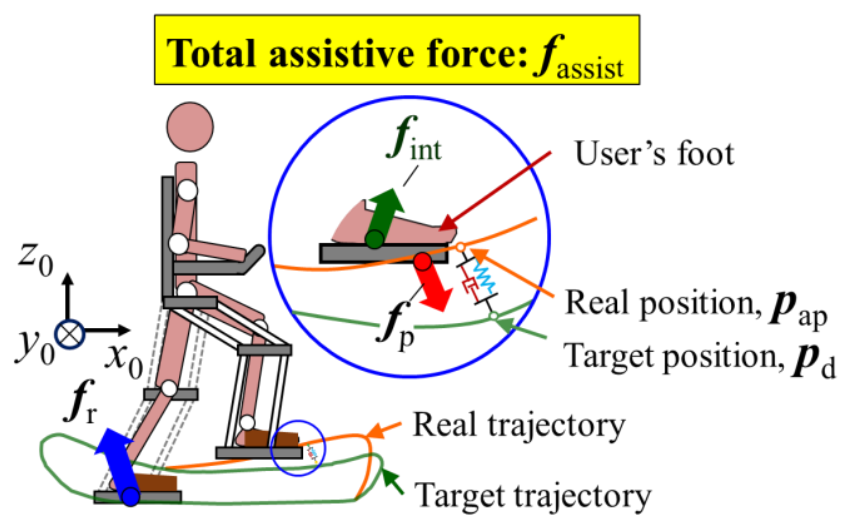

Fig. 5 Impedance control diagram.

To allow the feet of the apparatus to trace the target trajectory, we used the impedance control method to get the value of $f_{\mathrm{p}}$. A schematic diagram of impedance control is shown in Fig. 5. Impedance control expresses the relationship between position tracking errors and the interaction force $f_{\mathrm{p}}$ by a mass-spring-damper system. The system needs the position (velocity and acceleration) as an input and then produces a force to make the real foot position track the target trajectory. When the error between the target and the real position becomes larger, the force $f_{\mathrm{p}}$ also need to increase, which acts like a spring system. To ensure the smoothness of the process of tracking target trajectory, a damper system is also inquired. Thus, a virtual force that acts the sole of the foot was derived through Eq. (2). A certain torque is outputted by the apparatus to drive the system.

$$
\boldsymbol{f}_{\mathrm{p}}=M_{\mathrm{d}} \ddot{\boldsymbol{p}}_{\mathrm{ap}}+D_{\mathrm{d}} \dot{\boldsymbol{p}}_{\mathrm{e}}+K_{\mathrm{d}} \boldsymbol{p}_{\mathrm{e}} \in \mathrm{R}^{3}
$$

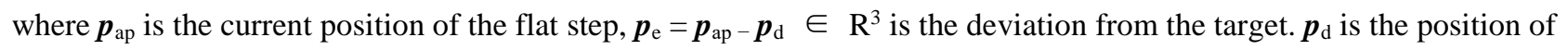
the target varying according to the walking phase of the trajectory,

$\boldsymbol{p}_{\text {ap }}=\left\{\begin{array}{lll}p_{\text {apx }} & p_{\text {apy }} \quad p_{\text {apz }}\end{array}\right\}^{\mathrm{T}} \in \mathrm{R}^{3}, \quad \boldsymbol{p}_{\mathrm{d}}=\left\{\begin{array}{lll}p_{\mathrm{dx}} & p_{\mathrm{dy}} & p_{\mathrm{dz}}\end{array}\right\}^{\mathrm{T}} \in \mathrm{R}^{3}$.

$M_{\mathrm{d}}, K_{\mathrm{d}}$, and $D_{\mathrm{d}}$ were determined from the previous research (Tanaka et al., 2012). $M_{\mathrm{d}}$ is the desired mass matrix when the user moves. $K_{\mathrm{d}}$ is the desired stiffness matrix when the user moves. $D_{\mathrm{d}}$ is the desired damping matrix when the user moves.

$M_{\mathrm{d}}=\operatorname{diag}\left[\begin{array}{lll}m_{\mathrm{x}} & m_{\mathrm{y}} & m_{\mathrm{z}}\end{array}\right] \in \mathrm{R}^{3 \times 3}, \quad K_{\mathrm{d}}=\operatorname{diag}\left[\begin{array}{lll}k_{\mathrm{x}} & k_{\mathrm{y}} & k_{\mathrm{z}}\end{array}\right] \in \mathrm{R}^{3 \times 3}, \quad D_{\mathrm{d}}=\operatorname{diag}\left[\begin{array}{lll}d_{\mathrm{x}} & d_{\mathrm{y}} & d_{\mathrm{z}}\end{array}\right] \in \mathrm{R}^{3 \times 3}$.

The foot of the user is secured in the sole of the assistive device by soft belts, which can be regarded as a linear constraint. The stiffness was determined by other researches (Taherifar et al., 2018). The lower limb of the device and leg of the user consist of a mechanism as shown in Fig. 6. Because the user's foot is restrained in a soft and elastic slipper, the relationship between the user and slipper is expressed as a spring-damper system in this paper. After the user's feet are restrained on the assistive device, the entire leg of the user can be driven by the motors mounted on the hip joint and the knee joint. This assistive device allows the user's lower limbs to move more freely within the frame than other devices that fix the user's thighs and calves. Therefore, there is a relative movement between the user's foots and the shoes on the device. After inputting the relative motion between the assistive device and the user, the interaction force between the assistive device and human was derived using Eq. (3).

$$
\boldsymbol{f}_{\text {int }}=K_{\mathrm{s}}\left(\boldsymbol{p}_{\text {ap }}-\boldsymbol{p}_{\mathrm{h}}\right)+D_{\mathrm{s}}\left(\dot{\boldsymbol{p}}_{\mathrm{ap}}-\dot{\boldsymbol{p}}_{\mathrm{h}}\right) \in \mathrm{R}^{3}
$$

Here, $f_{\text {int }}$ is a vector of the interaction force exerted by the exoskeleton on the user. $K_{\mathrm{s}}$ and $D_{\mathrm{s}}$ are determined by the stiffness and dissipation of the soft strap between the user and the assistive device. $\boldsymbol{p}_{\text {ap }}$ and $\boldsymbol{p}_{\mathrm{h}}$ are the foot positions of assistive device and human respectively.

$$
\boldsymbol{f}_{\text {int }}=\left\{f_{\text {intx }} \quad f_{\text {inty }} \quad f_{\text {intz }}\right\}^{\mathrm{T}} \in \mathrm{R}^{3}, \quad \boldsymbol{p}_{\mathrm{h}}=\left\{\begin{array}{lll}
p_{\mathrm{hx}} & p_{\text {hy }} \quad p_{\mathrm{hz}}
\end{array}\right\}^{\mathrm{T}} \in \mathrm{R}^{3} .
$$




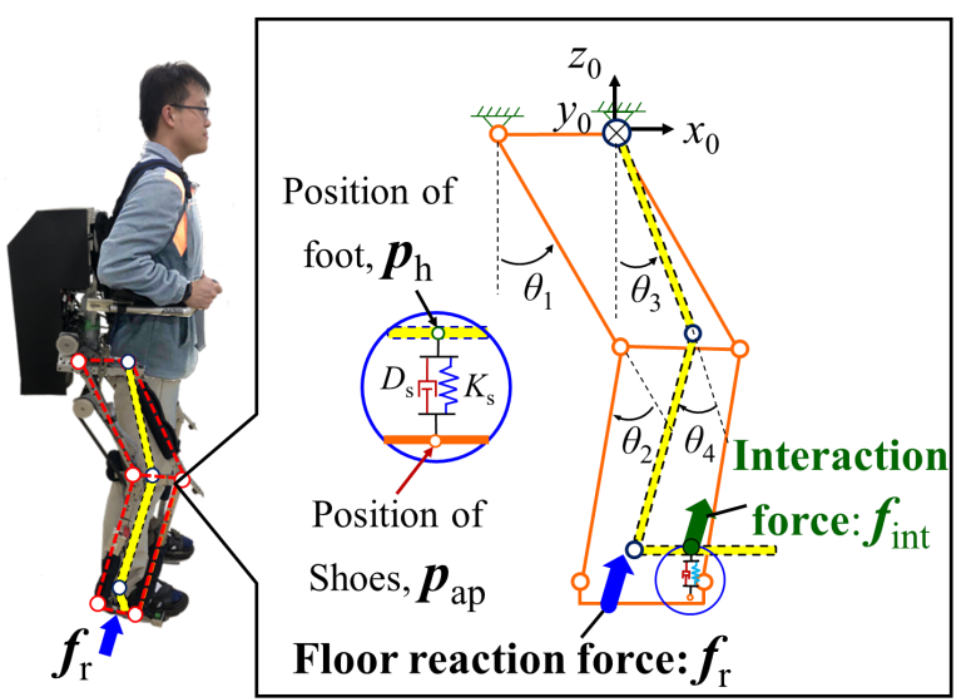

Fig. 6 Human model to calculate interaction force.

As shown in Fig. 5, the foot of the user, the footplate of the device, and the target trajectory contribute a system. To make the footplate trace the target trajectory, the device receives $f_{\mathrm{p}}$. Based on the interaction force $f_{\text {int }}$ between the user and the device, the assistive force of the user was derived from Eq. (4).

$$
f_{\text {assist }}=f_{\text {int }}+f_{\mathrm{p}} \in \mathrm{R}^{3}
$$

Here, $\boldsymbol{f}_{\text {assist }}=\left\{f_{\text {assistx }} \quad f_{\text {assisty }} \quad f_{\text {assistz }}\right\}^{\mathrm{T}} \in \mathbf{R}^{3}$.

The impedance control method is the basic control method of a walking assistive device. It is widely used in human-exoskeleton systems. By adjusting the output torque according to the error between the target trajectories and current foot positions, the feet of the device will move along the set trajectory. In the meantime, the user also performs the exercise. Therefore, using this device, the user can get over the effect of aging to some extent.

\section{Principle of posture compensation}

With the proper assistive force, the assistive device can help the user at a defined motion. Elderly people need to walk on stairs to achieve their daily living activities. Therefore, the purpose of the newly proposed compensation method that is to stabilize the user with device on stairs was proposed. Previously, the assistive device moved along target trajectories from healthy people (Tanaka et al., 2012). For a healthy elderly, he or she can be assisted using the trajectory. However, for a weakened elderly person, because his or her movement is very slow, the trajectories of a healthy person are very unsuitable for the user. Thus, in this section, we proposed a posture-compensated method to modify the walking motion of a healthy person to make it suitable for elderly people.

\subsection{Proposal of the posture-compensated control}

In the daily lives of the elderly, they need to walk in various conditions such as flat ground or stairs, and the motion in those conditions is different. The trajectories for various walking conditions are shown in Fig. 7. Each trajectory consists of 20 points. 0-9 points are the target position in stance phase. 10-19 points are the target position in swing phase. After inputting diverse trajectories, the apparatus can provide exercise to prevent muscle contraction on different terrains. Using the impedance control introduced in section 2, the device can assist along predefined trajectories. Besides, the trajectories for various terrains can be transferred automatically. The trajectories of the foot are viewed from the hip joint, which means that the hip joint is the origin point of the 2-D coordinate system. By modifying the target trajectories, the walking motion of the device can be adjusted to a stable motion for the elderly people. This is the basis of our proposed method, which is to compensate the posture of the device by adjusting the target trajectory (Yang et al., 2018a). 


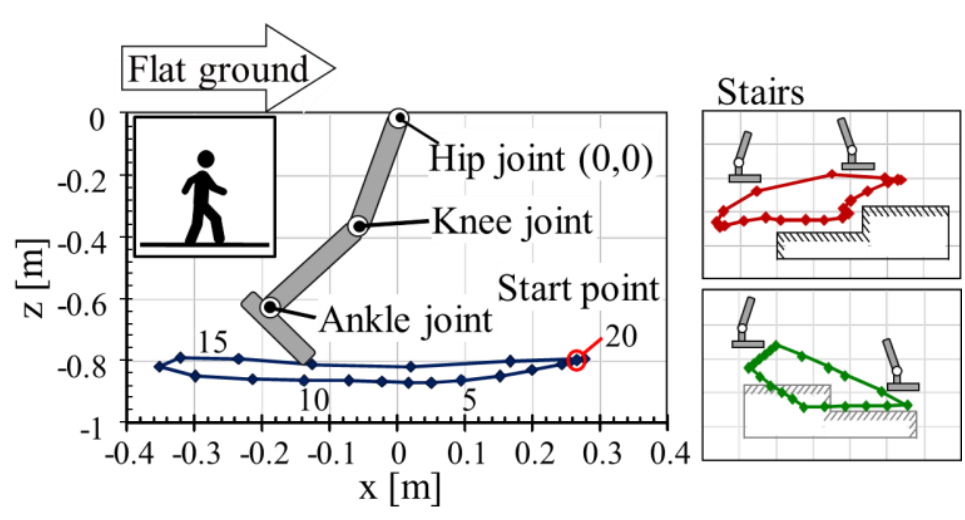

Fig. 7 Walking trajectories on various terrains.

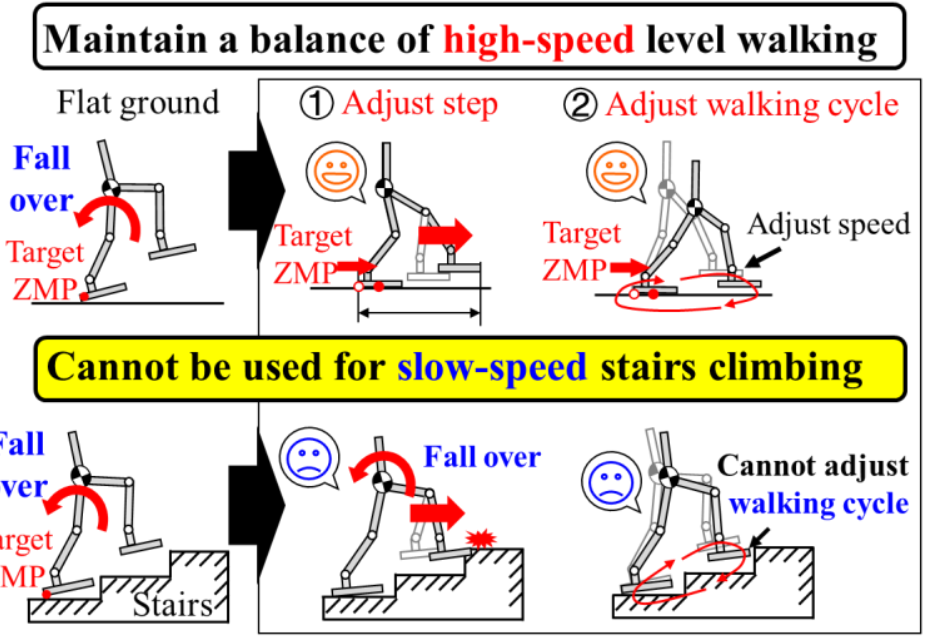

(a) Stability control methods of a humanoid robot

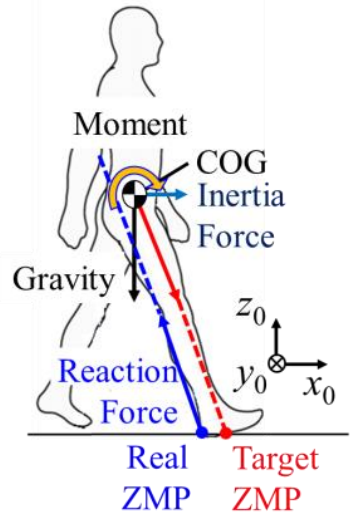

Fig. 8 Relationship of real and target ZMPs.

Fig. 9 Stability control methods of a humanoid robot.

The stability of bipedal walking is one of the most important issues in human walking. Since the 1970s, the zero-moment criterion, proposed as a stability index, has been widely used in the walking gait of bipedal robots (Vukobratovic and Borovac, 2004). The position of the ZMP has been utilized on many walking robots to judge whether the motion was stable. If the ZMP is in the supporting polygon, then the motion of the robot is considered stable. The ZMP can be divided into two types: the target ZMP and the real ZMP, as shown in Fig. 8. The target ZMP is regarded as the normal ZMP, which is the projection of gravity and the initial force on the ground. The real ZMP is the center of pressure of the foot (Sardain and Bessonnet, 2004). Therefore, it is also called the center of pressure (COP). The target ZMP can be gotten from the calculation. The location of the target ZMP is also easier to be imagined. Therefore, we considered the adjustment of the ZMP at first.

At present, some walking assistive devices utilize the stability method of a humanoid robot on flat ground because stability control was first considered for a humanoid robot. The main strategy of the control method can be divided into two forms: 1) tuning the step length and 2) tuning the walking cycle, which means adjusting the walking speed, as shown in Fig. 9(a). However, this method is available for flat ground, not stairs. The elderly cannot adjust the stride, and they walk very slowly. Therefore, this method is unsuitable for the target user. Additionally, this method is unsuitable for stairs. As for stairs, if the stride length is adjusted, the swing foot will collide with the stairs. Besides, because the walking motion on stairs is very slow, the walking cycle can also not be adjusted significantly. Therefore, the only thing we can adjust is the device posture.

As shown in Fig. 9(b), we proposed a method that only changes the location of the trunk to keep balance and shift ZMP. By compensating the trajectories of two feet the same distance, the trunk will move. More importantly, to maintain the distance between the swing foot and stance foot, the target positions of the stance foot and swing foot are changed at the same time and distance, and the position of the hip joint can be changed simultaneously. Thus, we can adjust the posture. 


\subsection{Judgment and improvement of instability 3.2.1 Stable area and target area of ZMP}

In the walking motion of a bipedal walking robot, the ZMP should always be maintained within the stable area of a convex polygon formed by the supporting foot. This is the stability criterion. The description of the stable areas in different areas is in Fig. 10.

The walking motion of elderly people differs from that of normal people. Their walking motion is slow, cautious, and unstable. Unlike healthy people, whose ZMP moves along a trajectory in the single-stance phase, the ZMP of those weakened people is kept within the area of the support polygon.

Thus, we defined a stable area in a single-support polygon, as shown in Fig. 10(a). At low speed, motion is very dangerous in single stance phase. Even there is a slight shaking at the upper body, the ZMP will move at the edge of the footprint, and the user will be easy to fall down to the ground. Therefore, we defined a target area for ZMP to keep balance. $p_{\mathrm{xb}}$ is the distance from the boundary to the centerline of the stable area.

Similarly, in the double-stance phase, the stable area and target area are shown in Fig. 10(b). Because the ZMP is kept under the back foot in the previous single-stance phase, the ZMP needs to transfer to the front foot in double stance phase, the user thus will become stable for the initial position of the next step. Otherwise, the device will become more likely to stumble because the ZMP is farther from the stance foot compared with the method without ZMP control. Therefore, the target area in the double-stance phase locates at the front foot.

In details, the posture compensation is to adjust the trajectory of the hip joint and trunk. If the controller finds that the ZMP is outside of the target area, the trunk of the assistive device will be shifted forward or backward to move the ZMP to the target area. The trajectories in Fig. 7 are trajectories of the foot viewed from the hip joint, which means that the hip joint is the origin point of the 2-D coordinate system. However, because the stance foot is stationary relative to the ground, the real motion is that of the hip joint as viewed from the stance foot. Generally, the trajectories of the foot and hip joint are in opposite directions as shown in right figures in Fig. 10. Because the target position of the foot is $\boldsymbol{p}_{\mathrm{d}}$, the target position of the hip joint is $-\boldsymbol{p}_{\mathrm{d}}$. By compensating the trajectories of the stance foot, the trajectories of the hip joint will be compensated the same distance in the opposite direction. In this research, we shifted the target trajectory of the foot to move upper body of the device.

Furthermore, after this process, the hip joint shifted $\sum p_{\text {comp }}$, which is the compensation distance. The initial target trajectory of the foot $\boldsymbol{p}_{\mathrm{d}}$ also shifted $\sum p_{\text {comp }}$. Therefore, we can get the final target trajectory of the device $\boldsymbol{p}_{\mathrm{d} \_ \text {comp }}$ that means the compensated trajectory.

Equations (5) denotes the relationship between the compensated trajectory and the initial target trajectory:

$$
p_{d \_c o m p x}(k)=p_{\mathrm{dx}}(k)-\sum_{n=0}^{k} p_{\text {comp }}(k)
$$

where $p_{d_{-} c o m p x}$ is the compensated target position in $\mathrm{x}$-direction. $p_{\mathrm{dx}}$ is the initial target position in the $\mathrm{x}$-direction. $p_{\text {comp }}$ is the compensation distance of each sample. $\Sigma p_{\text {comp }}$ is the constantly accumulating distance. $k$ is number of samples.

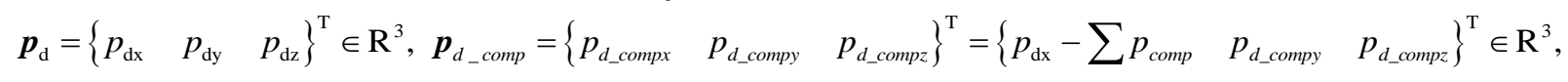

However, the compensation distance of each sample is different in single stance phase and double stance phase, as shown in Eqs. (6) and (7). The compensation distance in single stance phase is described in Eq. (6).

$$
p_{\text {comp }}(k)= \begin{cases}\alpha \frac{\left|p_{\mathrm{x}}\right|}{p_{\mathrm{x}}}\left[p_{\mathrm{dsx}}(k+1)-p_{\mathrm{dsx}}(k)\right], & p_{\mathrm{x}}<-p_{\mathrm{xb}}, p_{\mathrm{x}}>p_{\mathrm{xb}}, \\ 0, & -p_{\mathrm{xb}}<p_{\mathrm{x}}<p_{\mathrm{xb}} .\end{cases}
$$




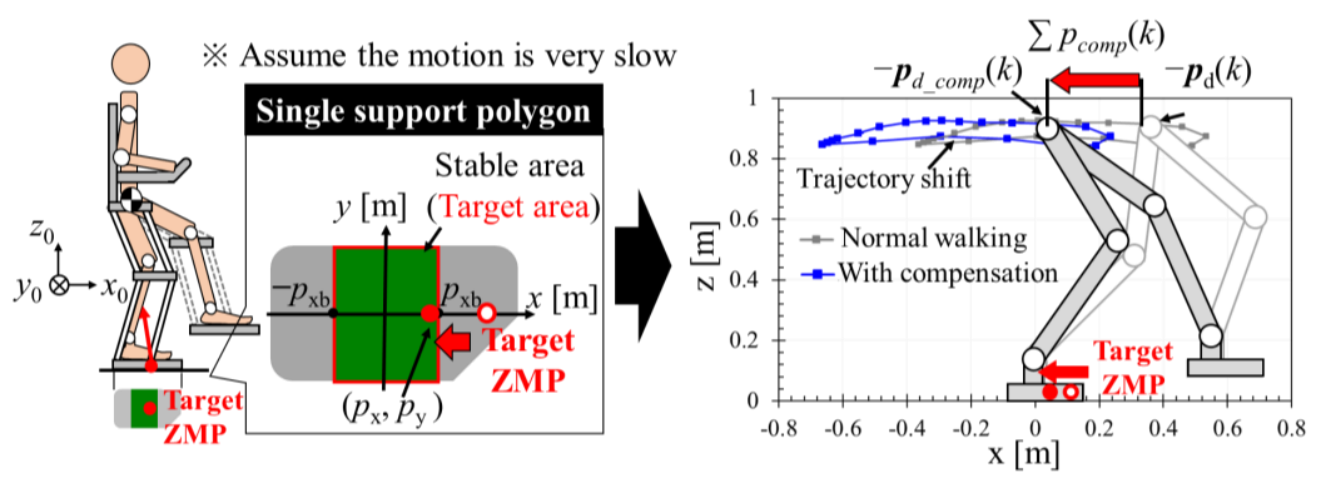

(a) Adjustment in single stance phase

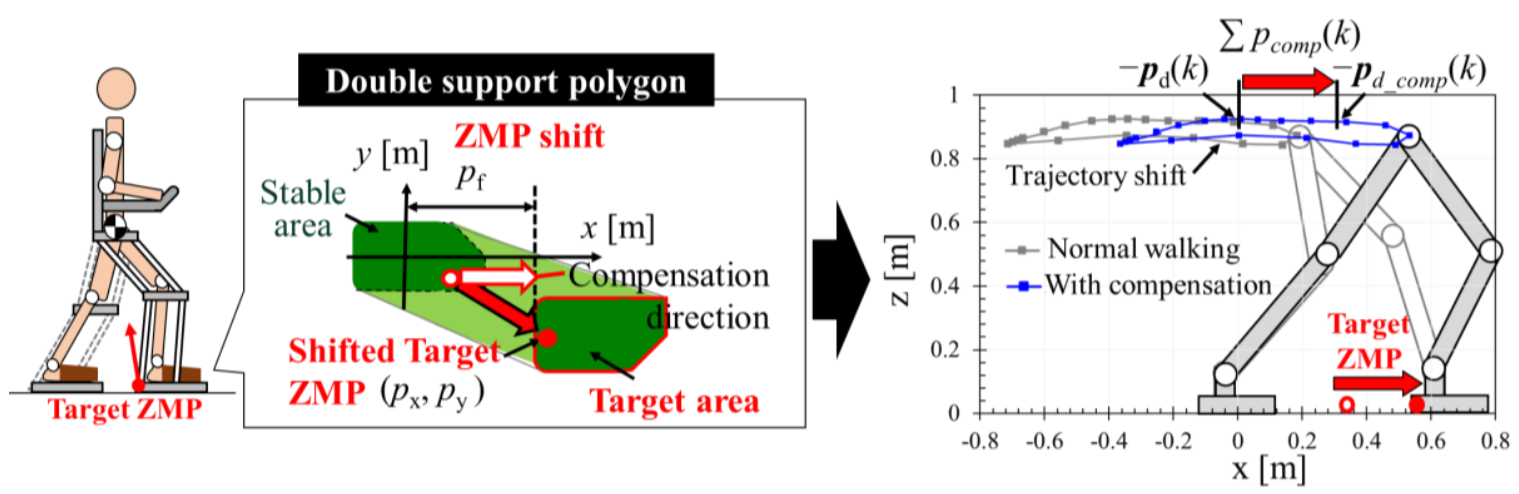

(b) Adjustment in double stance phase

Fig. 10 Adjustment of ZMP to the target area.

where $p_{\mathrm{x}}$ is the location of ZMP in the $\mathrm{x}$-direction. $p_{\mathrm{dsx}}$ is the target position of the stance foot in $\mathrm{x}$-direction. $\boldsymbol{p}_{\mathrm{ds}}$ that is

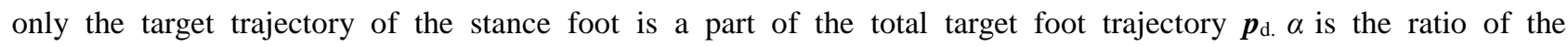
compensation distance of each sample, which was determined in experiment as 1.2 so that the compensation velocity is greater than that of the hip joint, and compensation can be achieved. $p_{\mathrm{xb}}$ is the distance from the boundary to the centerline of the stable area. The assistive device will compensate only if ZMP falls outside the stable area.

The compensation distance in double stance phase is described in Eq. (7).

$$
p_{\text {comp }}(k)= \begin{cases}\frac{l_{\text {stride }}}{T} T_{\mathrm{s}}, & p_{\mathrm{x}}<p_{\mathrm{f}}, \\ 0, & p_{\mathrm{x}} \geq p_{\mathrm{f}} .\end{cases}
$$

where $l_{\text {step }}$ denotes the step length. $T$ means the walking cycle that is set to $1.08 \mathrm{~s}$ as an average walking cycle. Because the ZMP need to be shifted from the back foot to front foot, the hip joint of the device also needs to move from one foot to another. We assume that the compensation speed of hip joint is the speed of the hip joint during normal walking. $T_{\mathrm{s}}$ denotes the sample time that is $0.001 \mathrm{~s}$.

\subsubsection{Motion of posture compensation}

The compensation motion in the single-stance and double stance phase is shown in Fig. 11. In the single-stance phase, the target area is the center area of the footplate. Similarly, in the double-stance phase, the stable area locates at the front foot. The ZMP transfer in the double-stance phase is also described. If it locates outside of the front foot, the hip joint will gradually move forward.

The relationship between the location of ZMP and the compensation distance are described. If the ZMP is in the target area, the device will not compensate. However, if the ZMP is in out of the target area, the total compensate distance will be added a small distance. By this method, the trajectory of the device was shifted continuously, and the original motion of the device was adjusted to a safer motion. 


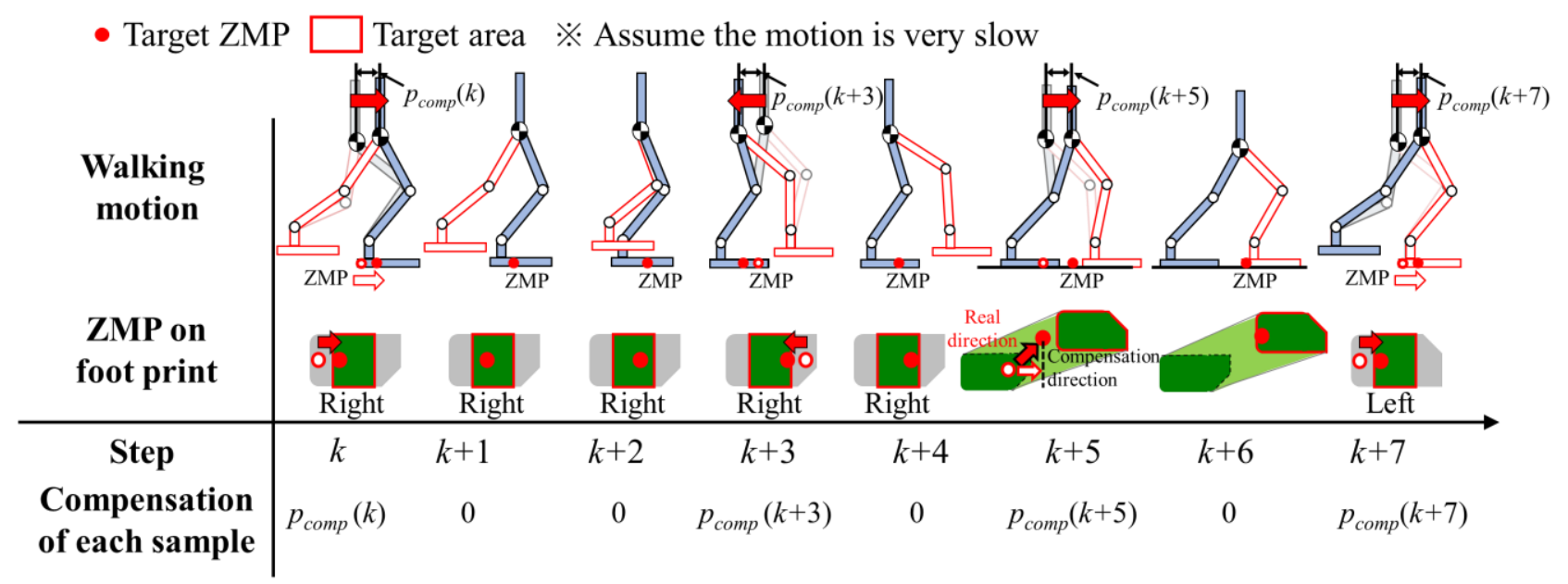

Fig. 11 Relationship of ZMP and compensation distance in each sample.

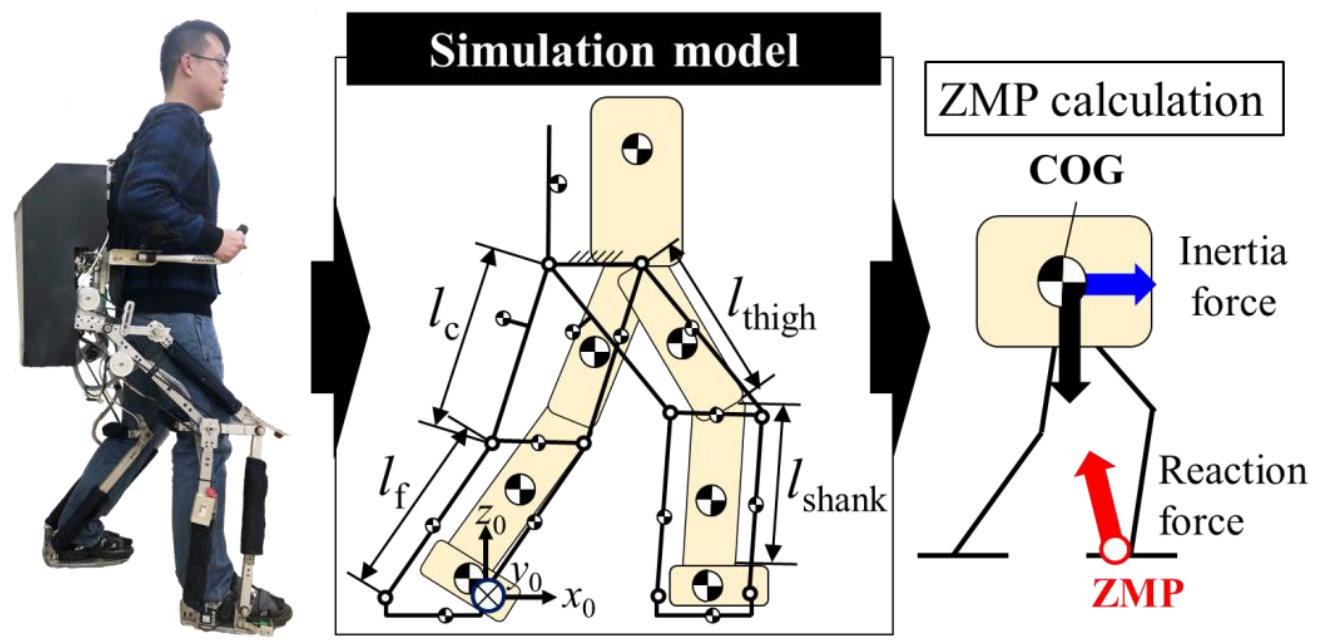

Fig. 12 Simulation model establishment and ZMP calculation.

\section{Study of simulation}

To evaluate the performance of the posture compensation method, the walking trajectories as shown in Fig. 7 were imported into the simulation model in Matlab ${ }^{\circledR}$ Simulink. Thus, the motion was simulated in three conditions: walking on flat ground, going up stairs, and going down stairs. The main purpose of this research was to verify the effectiveness of the stability method that will be used on the assistive device on stairs. However, we intended to first test this method on a flat road because walking directly on stairs is very dangerous.

In the simulation, we took $70 \mathrm{~kg}$ and $1.75 \mathrm{~m}$ as the weight and height of the user, respectively, to calculate the COG and ZMP, which were the same as those of the experimenter. The simulation was established as shown in Fig. 12. The location of ZMP is calculated from the COG of each link and part of the human-exoskeleton system.

The Simulink toolbox was applied to the model of the walking assistive device. After inputting the foot trajectory, the angles of each joint of the robot were obtained by inverse kinematics equations. Then, the angles of the user's legs were estimated base on angles of the device. Thus, the position of the COG can be calculated and used to derive the position of the ZMP.

\subsection{ZMP calculation method}

Usually, the ZMP is calculated from gravity and the acceleration of each element (Takanishi, 1993). However, because a walking robot is always a complicated system, its ZMP calculation amount is very large. Therefore, some simplification methods have been proposed to speed up the calculation. 


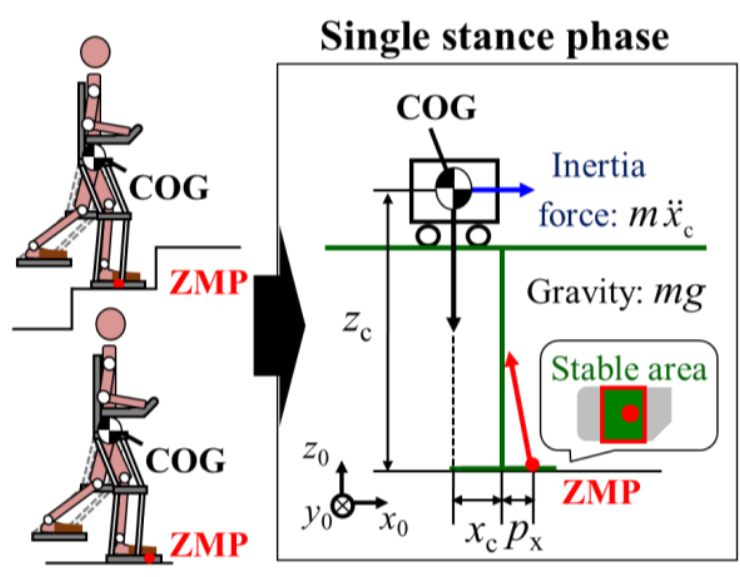

Fig. 13 Cart-table model to calculate ZMP.

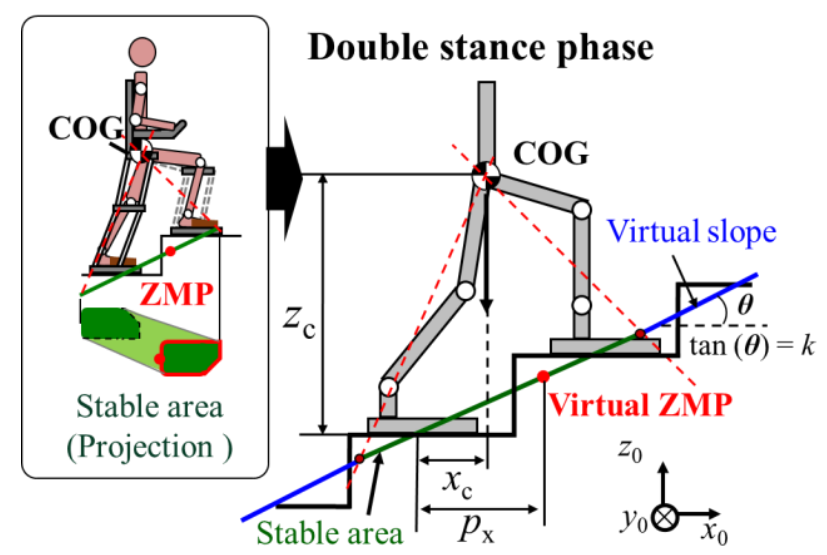

Fig. 14 Virtual slope model to calculate ZMP on stairs.

A proposed three-dimensional linear inverted pendulum mode was widely used. The method believes that the mass should move along an arbitrarily defined plane (Kuo et al., 2005). Naceur et al. (2017) and Chafroud et al. (2017) proposed a liquid level model as a simplified model for calculating the position of the ZMP by combining the ZMP approach and the inverted pendulum. The model considers the movement of the robot as a liquid moving on a flat road or slope. The speed of the movement causes a change in the liquid level. Based on the inverted pendulum model, Shimmyo et al. (2013) used a cart-table model to simplify the robot and assumed that the COG was constrained in a horizontal plane. The movement of the COG was also considered to be the movement of the cart on the table. Using these methods, the motion of each element of the robot is simplified as the motion of a whole, which helps save computing time.

To calculate the location of the ZMP, the position of the COG was calculated at the first. The position of the COG was derived from the human-assistive model in Fig. 12.

Then, we select the cart-table model to calculate the ZMP of our device because our device is a complicated human-exoskeleton system. Using this simplification method, the calculation amount of the ZMP will be less. We thus calculated the position of the ZMP using Eq. (8), derived from the cart-table model. There are many equations available for ZMP calculation, but this is the easiest one.

$$
p_{\mathrm{x}}=x_{\mathrm{c}}-\frac{z_{\mathrm{c}}-p_{\mathrm{z}}}{g+\ddot{z}_{\mathrm{c}}} \ddot{x}_{\mathrm{c}}
$$

Here, $\left(x_{\mathrm{c}}, z_{\mathrm{c}}\right),\left(p_{\mathrm{x}}, p_{\mathrm{z}}\right)$ and $g$ represent the positions of the COG, ZMP and the acceleration of the COG. The position of the COG can be calculated from the predefined foot trajectory.

However, the equation of the ZMP varies for different surroundings. When the device walks on a continuous plane such as flat ground or slope, we can use the traditional cart-table model to derive the location of ZMP. When the device walks on stairs, where the ground is not continuous, we cannot get the position of the ZMP in the double-stance phase because the feet of the device are in two separate planes. Therefore, the calculation of the ZMP is divided into two different conditions (Sato et al, 2011).

As for the device on flat ground or in the single-stance phase on stairs, the ZMP is defined on the surface of the ground, and the acceleration of the COG in the z-direction is very small. Therefore, we can ignore the acceleration in the z-direction. The walking human-exoskeleton system can be simplified into a running cart on a table. The mass of the whole human-exoskeleton system is concentrated on the cart. The mass of the table is neglected. Therefore, the movement of the COG of the human-exoskeleton system is regarded as the movement of the cart on the table. As shown in Fig. 13, the gravity of the cart locates at the edge of the table foot. If the cart is stationary, table is unstable. However, if the cart has proper acceleration, ZMP can keep in the table foot. The cart-table system is stable at this moment. Because the moment around ZMP must be zero, we have Eq. (9) and then can derive Eq. (10).

$$
\tau_{\mathrm{ZMP}}=m g\left(x_{\mathrm{c}}-p_{\mathrm{x}}\right)-m \ddot{x}_{\mathrm{c}} z_{\mathrm{c}}=0
$$




$$
p_{\mathrm{x}}=x_{\mathrm{c}}-\frac{z_{\mathrm{c}}}{g} \ddot{x}_{\mathrm{c}}
$$

However, for the double-stance phase on the stairs, the position of the ZMP cannot be calculated using the traditional methods. Therefore, we regard stairs as being a virtual slope (Sato et al, 2011), as shown in Fig. 14. The location of ZMP is described as Eq. (11). The virtual stable area in double stance phase can also be gotten according to the projection from COG to the footprints. During the simulation, we calculated the smallest support area as the stable area. The stable area of walking upstairs is from $-0.15 \mathrm{~m}$ to $0.65 \mathrm{~m}$. The stable area of descending stairs is from $-0.13 \mathrm{~m}$ to $0.67 \mathrm{~m}$. Totally, although the virtual slope method is inaccurate, the method can estimate ZMP in double stance phase. As a method to denote ZMP in simulation, it is available.

$$
p_{\mathrm{x}}=x_{\mathrm{c}}-\frac{z_{\mathrm{c}}-k p_{\mathrm{x}}}{g+\ddot{z}_{\mathrm{c}}} \ddot{x}_{\mathrm{c}}
$$

where $k$ is the gradient of the stairs. It can be deduced as Eq. (12).

$$
p_{\mathrm{x}}=\frac{\left(g+\ddot{z}_{\mathrm{c}}\right) x_{\mathrm{c}}-z_{\mathrm{c}} \ddot{x}_{\mathrm{c}}}{g+\ddot{z}_{\mathrm{c}}-k \ddot{x}_{\mathrm{c}}}
$$

By Eq. (10) or (12), the stability of the user with the device was described by comparing the ZMP position with the defined stable area. If the device was unstable, the waist of the robot then was adjusted forward or backward at the distance calculated by Eqs. (6) and (7) until the ZMP returned to stable area once more

\subsection{Optimal side length of stable area}

Because elderly people walk slowly, it is dangerous for them to walk in single stance phase. Thus, we proposed a method to keep the ZMP in a center area on foot plate, which can reduce the risk of falls when ZMP moves to the edge of foot. Because the disturbance that occur suddenly while walking can cause the instability, we wanted to find the best side length of the stable area. Using this side length, the assistive device can reduce the disturbance to the utmost extent. We found the best side length $(0.16 \mathrm{~m})$ through simulation. The procedure of the simulation is lists as follows:

The procedure is denoted in Fig. 15. Firstly, we added a disturbance signal (amplitude: $0.03 \mathrm{~m}$, frequency: $10 \mathrm{~Hz}$ ) that is closed to the essential tremor of human body to the trunk (Jankovic, 2008). Secondly, the proposed posture compensation method was used to stabilize the assistive device. Then, the amplitude of the disturbance after compensation was calculated. After that, we changed the side length of the stable area and did the previous steps again. Finally, we found that the side length of $0.16 \mathrm{~m}$ has the minimal disturbance as shown in Table 2. Therefore, we defined $0.16 \mathrm{~m}$ as the side length of the stable area.

\section{Add disturbance to trunk $>$ Posture compensation at various stable area}

Find the best stable area
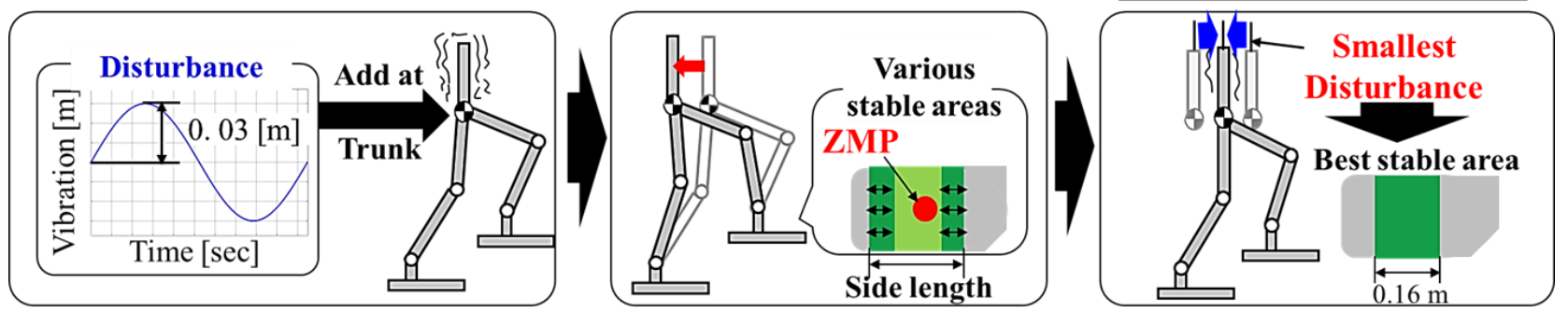

Fig. 15 Disturbance calculation to get the best stable area.

Table 2 Compensated vibration amplitude for different side length.

\begin{tabular}{c|c|c|c|c|c}
\hline \hline Side length [m] & 0.12 & 0.14 & 0.16 & 0.18 & 0.2 \\
\hline Compensated vibration amplitude $10^{-2}[\mathrm{~m}]$ & 2.04 & 2.18 & 1.77 & 2.26 & 2.45 \\
\hline
\end{tabular}




\subsection{Simulation results}

The simulation results are shown in Fig. 16. The green area represents the stable area that was introduced in Section 3. In the single-stance phase, the size is smaller; in the double-stance phase, it is larger.

From the figures, it can be seen that the walking motion is divided into two models. The blue curve of the ZMP always stays within the stable (green) area. This model is for a person who cannot walk fast. Therefore, they can walk more cautiously. The red curve sometimes moves outside of the stable area, which means that the walking is freer. This model is for healthy elderly people. Nevertheless, compared with flat ground walking, the ZMP movement distance in the double-stance phase is shorter because the stride length is shorter on stairs.

In the single-stance phase, the position of the ZMP is kept in the center area. Without compensation, the position of the ZMP will move outside of the stable area, which is proper for the healthy elderly. In the double-stance phase, we transfer the ZMP from back foot to the front foot. The ZMP thus moves forward. If there is no compensation, the position of the ZMP is invariable in this phase as time goes on.

Overall, regardless of the surroundings, the ZMP moves forward in one step because our COG moves forward relative to the support foot. Compared with the walking motion on flat ground, the error between walking with and without the ZMP control method is smaller on stairs. This is because the stride length on stairs is smaller. Among the three results, the gap of the ZMP trajectories for walking downstairs is the smallest because the initial posture of one step of walking downstairs is stable.

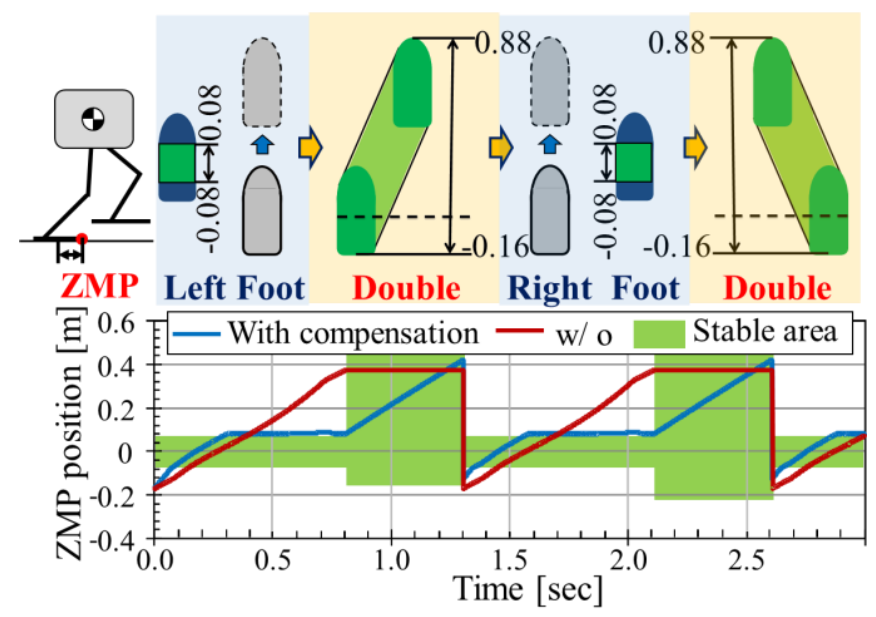

(a) Results of level walking

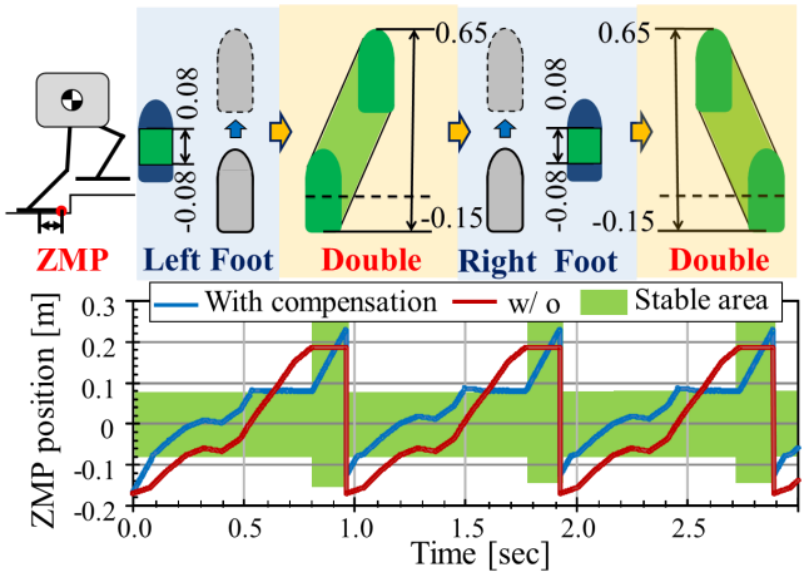

(b) Results of walking upstairs

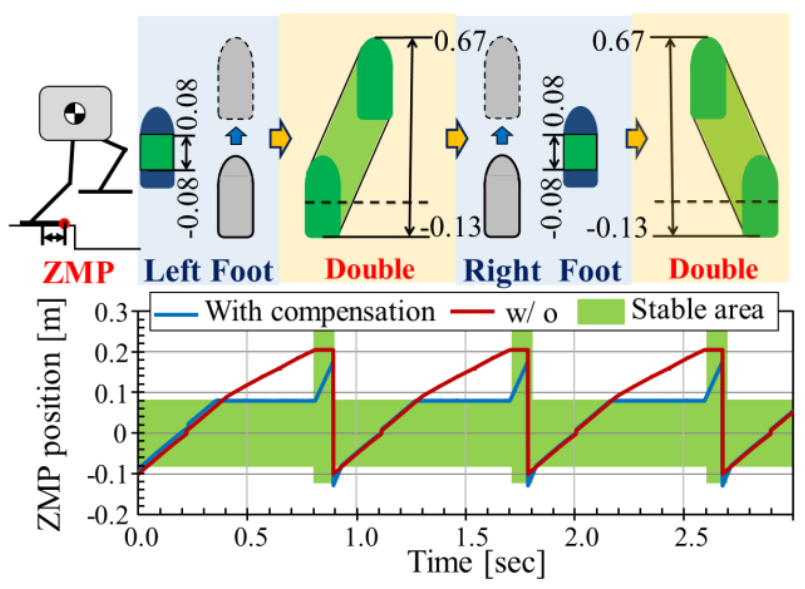

(c) Results of walking down stairs

Fig. 16 Simulation results in various conditions. 


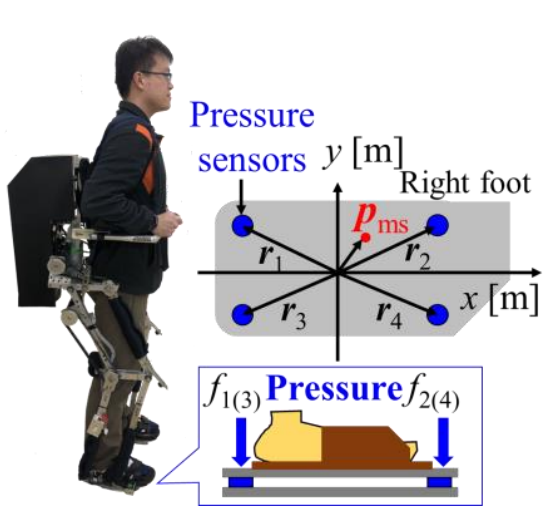

(a) Single-stance phase

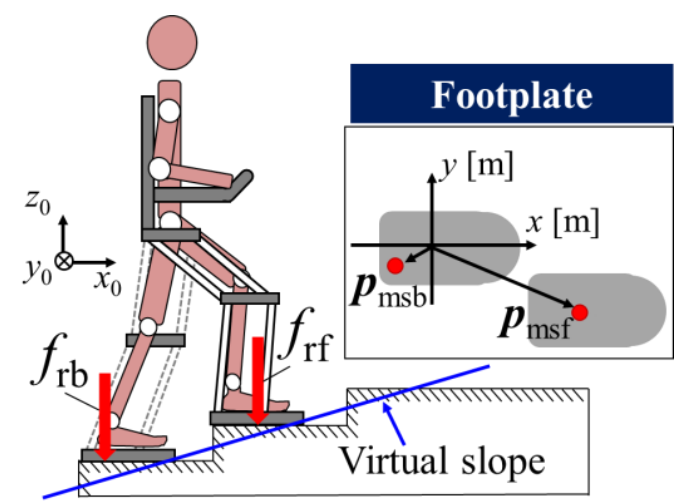

(b) Double-stance phase

Fig. 17 Measurement of ZMP.

\section{Validation experiments}

Some experiments were performed to verify the effectiveness of our proposed method. As described in the simulation, the height of the user was $1.75 \mathrm{~m}$, and the weight of the user was $70 \mathrm{~kg}$. We did the experiment corresponding to level walking, walking upstairs, and walking downstairs.

Unlike the simulation, we did not use the cart-table formula to calculate the position of the ZMP. Because the amount of the calculation was too large, the method was not suitable for real-time control, especially for the human-exoskeleton system, which is nonlinear and very complex. Therefore, we used the system introduced in Fig. 3 to get the position of the ZMP.

\subsection{Measurement of ZMP}

The target ZMP is calculated from the angle of each joint. The position of the real ZMP is measured from the values of the pressure sensors under the footplate. Four pressure sensors are distributed under the shoes of the apparatus. According to the respective pressure values, the apparatus can calculate the center of pressure (Yang et al., 2018b). The real ZMP is easy to calculate. Consequently, in the simulation, we control the target ZMP calculated from the angle of each joint. In the experiments, we used the ZMP measured from the pressure sensors as a stability standard because the calculation time was less, which meant that the calculation cost was less.

The location of the four pressure sensors in the footplate is shown in Fig. 17(a). We derived the position of the ZMP in the single-stance phase using Eq. (12).

$$
\boldsymbol{p}_{\mathrm{ms}}=\frac{\sum_{i=1}^{4} f_{i} \boldsymbol{r}_{i}}{\sum_{i=1}^{4} f_{i}} \in \mathrm{R}^{3}
$$

Here, $\boldsymbol{p}_{\mathrm{ms}}$ is the measured ZMP in the single-stance phase, $f_{i}$ is the pressure of each sensor. $\boldsymbol{r}_{i}$ is the vector from the original point in the center of the footplate to each sensor. $\boldsymbol{p}_{\mathrm{ms}}$ and $\boldsymbol{r}_{i}$ are two-dimensional.

$$
\boldsymbol{p}_{\mathrm{ms}}=\left\{\begin{array}{lll}
p_{\mathrm{msx}} & p_{\mathrm{msy}} & p_{\mathrm{msz}}
\end{array}\right\}^{\mathrm{T}}=\left\{\begin{array}{lll}
p_{\mathrm{msx}} & p_{\text {msy }} & 0
\end{array}\right\}^{\mathrm{T}} \in \mathrm{R}^{3}, \quad \boldsymbol{r}_{i}=\left\{\begin{array}{lll}
r_{i x} & r_{i y} & r_{i z}
\end{array}\right\}^{\mathrm{T}}=\left\{\begin{array}{lll}
r_{i x} & r_{i y} & 0
\end{array}\right\}^{\mathrm{T}} \in \mathrm{R}^{3} .
$$

During the double-stance phase, the position of the COP of complete walking assistance is calculated from the COP of each foot, as shown in Fig. 17(b) and Eq. (13).

$$
\boldsymbol{p}_{\mathrm{md}}=\frac{f_{\mathrm{rf}} \boldsymbol{p}_{\mathrm{msf}}+f_{\mathrm{rb}} \boldsymbol{p}_{\mathrm{msb}}}{f_{\mathrm{rf}}+f_{\mathrm{rb}}} \in \mathrm{R}^{3}
$$

Here, $\boldsymbol{p}_{\mathrm{md}}$ is the measured ZMP in double stance phase. $f_{\mathrm{rf}}$ and $f_{\mathrm{rb}}$ are the pressures of the front foot and back foot, respectively. $\boldsymbol{p}_{\mathrm{msf}}$ and $\boldsymbol{p}_{\mathrm{msb}}$ are the COP of the front foot and the rear foot, respectively. 


$$
\begin{aligned}
& \boldsymbol{p}_{\mathrm{md}}=\left\{\begin{array}{lll}
p_{\mathrm{mdx}} & p_{\mathrm{mdy}} & p_{\mathrm{mdz}}
\end{array}\right\}^{\mathrm{T}}=\left\{\begin{array}{lll}
p_{\mathrm{mdx}} & p_{\text {mdy }} & 0
\end{array}\right\}^{\mathrm{T}} \in \mathrm{R}^{3}, \boldsymbol{p}_{\text {msf }}=\left\{\begin{array}{lll}
p_{\text {msfx }} & p_{\text {msfy }} & p_{\text {msfz }}
\end{array}\right\}^{\mathrm{T}}=\left\{\begin{array}{lll}
p_{\text {msfx }} & p_{\text {msfy }} & 0
\end{array}\right\}^{\mathrm{T}} \in \mathrm{R}^{3}, \\
& \boldsymbol{p}_{\mathrm{msb}}=\left\{\begin{array}{llll}
p_{\mathrm{msbx}} & p_{\text {msby }} & p_{\text {msbz }}
\end{array}\right\}^{\mathrm{T}}=\left\{\begin{array}{lll}
p_{\text {msbx }} & p_{\text {msby }} & 0
\end{array}\right\}^{\mathrm{T}} \in \mathrm{R}^{3} .
\end{aligned}
$$

\subsection{Experiments of posture compensation in various phases}

To verify our proposed method, we did experiments in various surroundings. Because walking on the stairs is very dangerous, we first did experiments on flat ground. We collected a total of three sets of experimental data. In each set of experiments, the assistive device was commanded to walk six steps. We experimented with and without assistance. Finally, we compared the ZMP situation under two conditions. The stride length was set to $1.25 \mathrm{~m}$, and the walking cycle was $2.4 \mathrm{~s}$. We finally chose four steps that had clear results. They are shown in Fig. 18. We utilized the Butterworth filter to exclude the noise of the experimental results. The cutoff frequency of the low pass filter is $0.5 \mathrm{~Hz}$. The order of the filter is 1 .

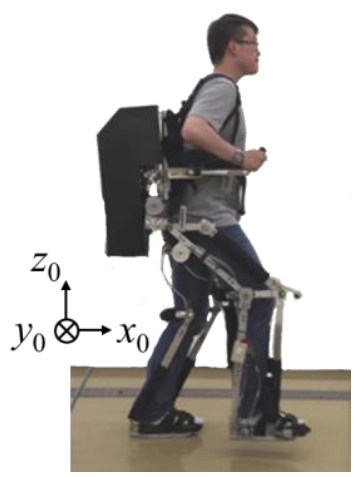

(a) Walking motion on flat ground

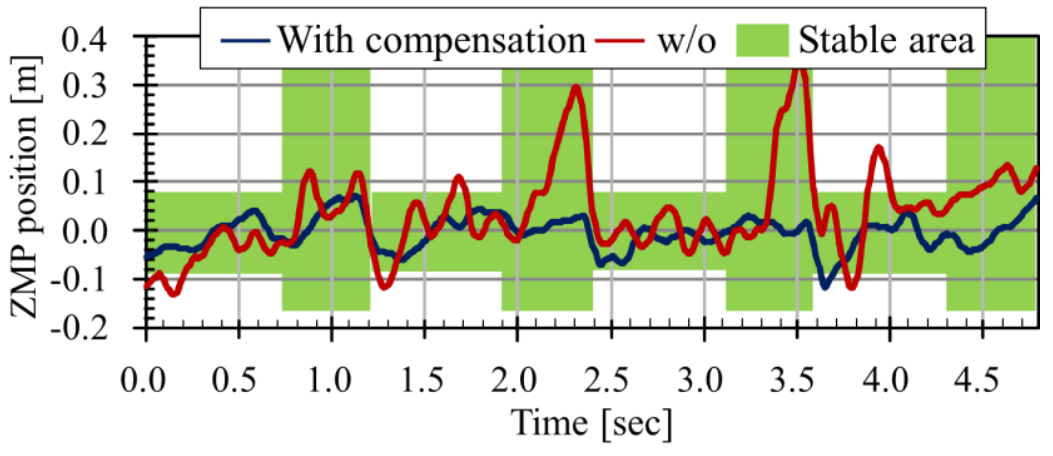

(b) ZMP Comparison in the $\mathrm{x}$-direction of level walking

Fig. 18 ZMP experiment results of level walking.

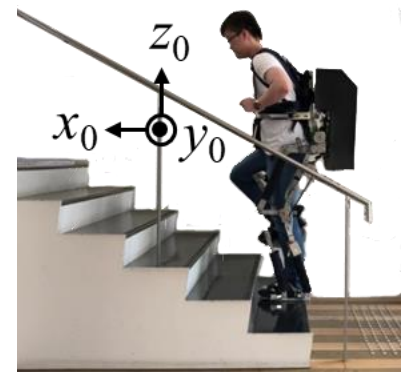

(a) Walking motion on flat ground

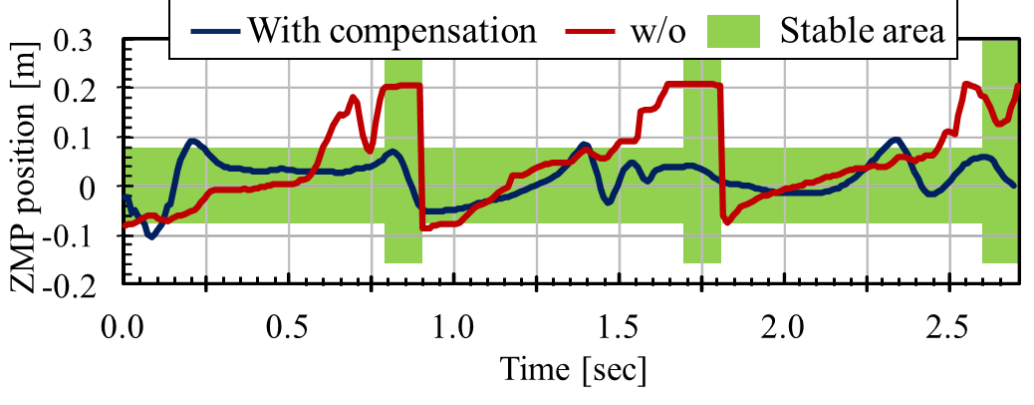

(b) ZMP Comparison of in the X-direction upstairs-walking

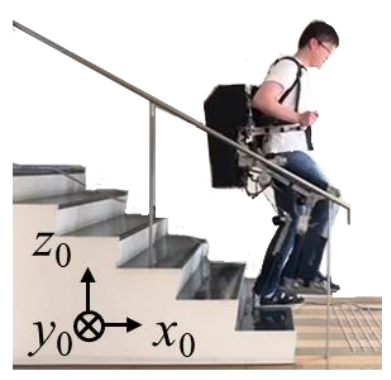

(a) Walking motion on flat ground

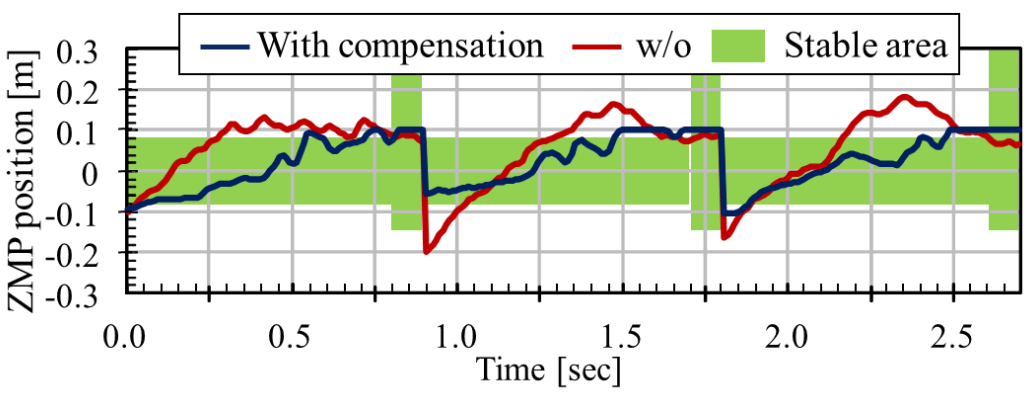

(b) ZMP Comparison in the x-direction of downstairs-walking

Fig. 20 ZMP experiment results of walking downstairs. 
From the results in Fig. 18, the ZMP of the human-exoskeleton moves forward in one step. With or without the compensation method, the ZMP can basically remain in the stable area, which is caused by the subject. The subject was a healthy person who could keep balance by himself.

However, by comparing the two curves, we see that the range of the ZMP after compensation was effectively reduced to the stable (green) area. This phenomenon means that the walking motion of the user becomes more cautious. The device can assist the user to walk more stably with our proposed method. The assistive device thus has two walking modes. One is for healthy people who walk quickly, who use the device to do exercise and prevent atrophy. This kind of user can walk independently and has healthy body conditions. Without ZMP compensation, they can get more active exercise. The other model is for weak users, who need our proposed method to prevent falls. Our method can reduce the risk of stumbling to some extent.

After verifying the effectiveness of our proposed method on flat ground, we did experiments on stairs. Stabilizing the walking motion on stairs is our main target. We did experiments on indoor stairs with height was $0.16 \mathrm{~m}$ and width of $0.32 \mathrm{~m}$. We did three groups of experiments to verify the method. In each group of experiments, the subject was asked to walk five steps with and without the posture compensation method. The stride length was set to $0.62 \mathrm{~m}$, and the walking cycle was $1.2 \mathrm{~s}$. We finally chose three steps that had clear results. The experiment results are shown in Figs. 19 and 20.

From the figures, the ZMP generally rises in one step for both walking up and downstairs. Besides, there are some disturbances when the user walked upstairs. Comparing the range of the ZMP with and without our proposed method, we can see that it was effectively controlled in the predefined area after compensation. Therefore, the motion is more cautious. Comparing the results of walking on flat ground and stairs, the range of the ZMP is smaller if the subject walked on stairs. This is because the stride length of stair walking is shorter. All in all, with our proposed method, the walking motion can be transformed into a motion that is cautious and suitable for some users with poor health conditions.

\section{Conclusion}

In this paper, we developed a new posture-compensated method to shift ZMP that can be used on stairs. Because the distance between every two stairs is constant, most ZMP control methods are not feasible for stairs. We, therefore, proposed a new compensation method that maintained the distance of both feet. It has better prospects. It can be used not only on stairs but also on flat ground and slopes.

First, we chose the real ZMP, which is the COP, as our criterion of stability because the calculation cost was small and accurate. The ZMP is limited to a stable area in various terrains. In the single-stance phase, the stable area is a central area of the footplate. In the double-stance phase on flat ground, the stable area is the area between the user's two feet. Also, in the double-phase of stairs, the stable area is a virtual slope. Then, we defined a posture compensation method that shifted the position of the hip joint. The compensation distance is decided by the distance between the ZMP and the border of the center area in the walking cycle. Next, we established a simulation system in Matlab ${ }^{\circledR}$ Simulink to test the validity of the posture compensation method on different terrains. We adjusted the parameters and filters during the simulation to make the experiments safer and save experiment time. Finally, we did the experiment on various terrains and achieved ZMP compensation. The proposed posture compensation method makes each motion safe and is suitable for elderly people.

\section{References}

Aphiratsakun, N. and Parnichkun, M., Balancing control of AIT leg exoskeleton using ZMP based FLC, International Journal of Advanced Robotic Systems, Vol.6, No.4 (2009), pp.319-328.

Chafroud, N., Haddad, N. and Braiek, N., Stable walking of humanoid robot based on a liquid level model, International Conference on Advanced Systems and Electric Technologies, (2017), pp.360-365.

Hirano, S., Saitoh, E., Tanabe, S., Katoh, M., Shimizu, Y., Yatsuya, K., Tanaka, H., Kagaya, H., Ishihara, K. and Uno, A., Comparison between gait-assisting robot (WPAL) and bilateral knee-ankle-foot orthoses with a medial single hip joint in gait reconstruction for patients with paraplegia, Japanese Journal of Comprehensive Rehabilitation Science, Vol. 6, (2015), pp.21-26. 
Jankovic J., Parkinson's disease: clinical features and diagnosis, Journal of Neurology, Neurosurgery, and Psychiatry, (2008), pp.368-376.

Kerrigan, D. C., Todd, M. and Della, C., Gender differences in joint biomechanics during walking, Am J Phys Med Rehabil, Vol.77, No.1, (1998), pp.2-7.

Kuo, A.D., Donelan, J.M. and Ruina, A., Energetic consequences of walking like an inverted pendulum: step-to-step transitions. Exercise and Sport Sciences Reviews, Vol.33, No.2, (2005), pp.88-97.

Low, K. H., Liu, X., Goh, C. H. and Yu H., Locomotive control of a wearable lower exoskeleton for walking enhancement, Journal of Vibration and Control, Vol.12, No.12, (2006), pp.1311-1336.

Naceur, C., Nahla, H. and Naceur, B., Stable Walking of Humanoid Robot Based on a Liquid Level Model, 2017 International Conference on Advanced Systems and Electric Technologies, (2017), pp.360-365.

Nakamura, R., Saito, H. and Nagasaki, H., Fundamental Kinematics (2003), pp. 334-335, Ishiyaku publishers Inc, 6th Edition. (In Japanese).

Reinkensmeyer, D. J., Aoyagi, D., Emken, J. L., Galvez, J. A., Ichinose, W., Kerdanyan, G., Maneekobkunwong, S., Minakata, K., Nessler, J. A., Weber, R., Roy, R. R., Leon, R., Bobrow, J. E., Harkema, S. J. and Edgerton, V. R., Tools for understanding and optimizing robotic gait training, The Journal of Rehabilitation Research and Development, Vol.43, No.5, (2006), pp.657-670.

Sakurai, T., Development of ZMP controls for walking reconstruction and walking assist for the elderly, Master Thesis, Shibaura Institute of Technology Graduate School, (2012) (In Japanese).

Sardain, P. and Bessonnet, G., Zero moment point-measurements from a human walker wearing robot feet as shoes, IEEE Transactions on Systems, Man, and Cybernetics-Part A: Systems and Humans, Vol. 34, No, 5, (2004) 638-648.

Sato, T., Sakaino, S., Ohashi, E. and Ohnishi, K., Walking trajectories planning on stairs using virtual slope for biped robots, IEEE Transactions on Industrial Electronics, Vol.58, No.4, (2011), pp.1385-1396.

Shimmyo, S., Sato, T. and Ohnishi, K., Biped walking pattern generation by using preview control based on three-mass model, IEEE Transactions on Industrial Electronics, Vol.60, No.11, (2013), pp.5137-5147.

Suzuki, K., Mito, G., Kawamoto, H., Hasegawa, Y. and Sankai, Y., Intention-based walking support for paraplegia patients with robot suit HAL, Adv. Robot, Vol.21, (2007), pp.1441-1469.

Taherifar, A., Vossoughi, G. and Ghafari, A. S., Assistive-compliant control of wearable robots for partially disabled individuals, Control Engineering Practice, Vol. 74, (2018), pp.177-190.

Takanishi, A., Biped walking robot compensating moment by trunk motion, Journal of the Robotics Society of Japan, Vol. 11, No. 3, (1993), pp.348-353 (In Japanese).

Talaty, M., Esquenazi, A. and Briceño, J. E., Differentiating ability in users of the ReWalk ${ }^{\mathrm{TM}}$ powered exoskeleton: an analysis of walking kinematics, 2013 IEEE 13th International Conference on Rehabilitation Robotics (ICORR), (2013), pp.1-5.

Tanaka, E., Ikehara, T., Sato, Y., Yusa, H., Ito, K., Saegusa, S., Nakagawa, K., Aokage, Y. and Yuge, L., Development of a walking assistance apparatus without fixation on legs and study on the assistance effectiveness with electromyography, Transactions of the Japan Society of Mechanical Engineers, Series C, Vol.77, No.775, (2011), pp.1119-1132 (In Japanese).

Tanaka, E., Ikehara, T., Yusa, H., Sato, Y., Sakurai, T., Saegusa, S., Ito, K. and Yuge, L., Walking-assistance apparatus as a next-generation vehicle and movable neuro-rehabilitation training appliance. Journal of Robotics and Mechatronics, Vol.24, No.5, (2012), pp.851-865.

Tanaka, E., Suzuki, T., Saegusa, S. and Yuge, L., Walking assistance apparatus able to select the control method according to the purpose of the user, World Automation Congress, (2014), pp.537-542.

Vukobratovic, M. and Borovac, B., Zero-moment point - thirty five years of its life, I. J. Humanoid Robotics, Vol.1, (2004), pp.157-173.

Yang, B. R., Lee, H. H. and Tanaka, E., Posture compensated zero-moment point control method of the walking assistance apparatus, ROBOMECH 2018 the Robotics and Mechatronics Conference (2018a), 2A2-D06.

Yang, B. R., Lee, H. H. and Tanaka, E., Stable posture compensation based on zero-moment point control method for a walking assistance apparatus, 2018 International Conference on Cyborg and Bionic Systems (2018b), pp.286-291. 